\title{
Graphical Affine Algebra
}

\author{
Filippo Bonchi \\ Universitá di Pisa \\ Pisa, Italy
}

\author{
Robin Piedeleu \\ University College London \\ London, United Kingdom
}

\author{
Pawel Sobocinski \\ University of Southampton \\ Southampton, United Kingdom
}

\author{
Fabio Zanasi \\ University College London \\ London, United Kingdom
}

\begin{abstract}
Graphical linear algebra is a diagrammatic language allowing to reason compositionally about different types of linear computing devices. In this paper, we extend this formalism with a connector for affine behaviour. The extension, which we call graphical affine algebra, is simple but remarkably powerful: it can model systems with richer patterns of behaviour such as mutual exclusion-with modules over the natural numbers as semantic domain - or non-passive electrical components-when considering modules over a certain field. Our main technical contribution is a complete axiomatisation for graphical affine algebra over these two interpretations. We also show, as case studies, how graphical affine algebra captures electrical circuits and the calculus of stateless connectors-a coordination language for distributed systems.
\end{abstract}

\section{INTRODUCTION}

Concurrent programming can be seen as the marriage of parallelism with synchronisation mechanisms. One of the earliest and most influential synchronisation mechanism is mutual exclusion [1], with the same underlying idea present in modern concurrent programming through hardware-assisted atomic constructs such as compare-and-set (CAS). Any theory that takes up Abramsky's challenge [2] to identify the fundamental structures of concurrency ought to be expressive enough to account for such fundamental synchronisation patterns. The remit of this paper is the development of a principled, compositional account of these fundamental structures of concurrency by characterising the underlying mathematical concept—affine relations-and exploring (somewhat unexpectedly!) related applications, notably non-passive electrical circuits.

\section{A. Graphical Linear Algebra (GLA)}

GLA [3], [4] is a diagrammatic language used to reason compositionally about different types of linear computing devices. String diagrams of GLA are recursively defined by sequential and parallel composition of the following basic operation, parametrised by a given semiring $\mathrm{R}$.

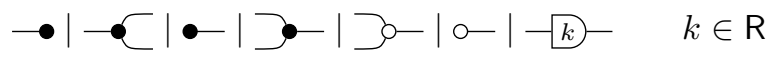

Here $\bigcirc-$ is addition, $\circ$ - the constant zero, $-k$ - is multiplication by $k, \multimap$ copy, $\longrightarrow$ discard, while $?-$ and $\bullet$ are the same operations right-to-left. This semantics is formalised via a recursively defined mapping of diagrams to relations over R-vectors: thus right-to-left operations are simply denoted by the opposite relations of their left-to-right cousins.

GLA has some claims of being fundamental. For $R=\mathbb{Z} / 2$, it is the syntax of the phase-free ZX-calculus [5], a simple algebra for pure state qubit computation [6]. For R the field
$\mathbb{R}(x)$ of polynomial fractions, it has been used to model linear dynamical systems [7]-[9], which itself goes back to Shannon [10] and the class of signal flow graphs [11], [12]. More recently [13] we showed that, by letting $R$ be the semiring $\mathbb{N}$ of natural numbers, GLA gives a compositional account of the behaviours of Petri nets. Indeed, the lack of additive inverses in $\mathbb{N}$ is well-suited to situations-as in classic Petri nets-where it is unreasonable to consider "negative resources".

Thus in different computational interpretations-from quantum and control-theoretic to concurrent-the set (1) of syntactic primitives and the specification of their relational behaviour remains the same. What changes is the denotational domain, that is, the kinds of relations that are characterised. GLA over a field (e.g. $\mathbb{Z} / 2, \mathbb{R}$ or $\mathbb{R}(x)$ ), equipped with the equations of interacting Hopf algebras, axiomatises linear relations (relations that are linear subspaces) [3], [4]. GLA over $\mathbb{N}$, equipped with the equations of the algebra of resources, axiomatises additive relations (relations that are $\mathbb{N}$-semimodules, containing the zero vector and closed under addition) [13].

\section{B. Missing Expressivity: Affine Relations}

Within the concurrent interpretation, mere additive relations are not quite powerful enough to capture essential behaviour patterns such as mutual exclusion. Indeed, consider the following idealised mutual exclusion connector, as considered in the calculus of stateless connectors [14].

$$
\text { O+ }
$$

The legal behaviours, as an $\mathbb{N}$-relation, is the finite set

$$
\left\{\left(\left(\begin{array}{l}
0 \\
0
\end{array}\right), 0\right),\left(\left(\begin{array}{l}
1 \\
0
\end{array}\right), 1\right),\left(\left(\begin{array}{l}
0 \\
1
\end{array}\right), 1\right)\right\}
$$

indicating that only one of the two inputs can synchronise with the output at any one time. The relation is not additive: e.g. $\left(\left(\begin{array}{l}1 \\ 0\end{array}\right), 1\right)+\left(\left(\begin{array}{l}0 \\ 1\end{array}\right), 1\right)=\left(\left(\begin{array}{l}1 \\ 1\end{array}\right), 2\right)$ is not included. We shall see, however, that it is an $\mathbb{N}$-affine relation. Moving from additive to affine relations expands the relational universe. For example, the empty relation $\varnothing$ is affine, but not additive.

The concept of affinity is of course better known over fields (e.g. $\mathbb{R}$ ) and is a mathematical playground of affine and convex geometry. It turns out that moving to affine relations is fruitful also in this context, namely in modelling electrical circuits. GLA has already been used to define a compositional semantics for passive linear circuits [15], that is, electrical circuits built exclusively from resistors, inductors and capacitors. The advantage of this approach is that it 
provides a rigorous setting in which to perform open network analysis purely diagrammatically.

Non-passive components, however, e.g. voltage and current sources are not linear but affine. For example, a $k$ volt source constrains the voltage $\left(\phi_{1}, \phi_{2}\right)$ and current $(i)$ pairs to be the following relation:

$$
\left.\stackrel{-}{\mathrm{k}} \longmapsto\left\{\left(\begin{array}{c}
\phi_{1} \\
i
\end{array}\right),\left(\begin{array}{c}
\phi_{2} \\
i
\end{array}\right)\right) \mid \phi_{2}-\phi_{1}=k\right\}
$$

Our motivating questions for the results presented in this paper are, therefore, (i) can the syntax of GLA be extended in a simple and principled fashion to capture $\mathbb{R}$-affine and $\mathbb{N}$ affine relations? And, if so, (ii) can we give an equational characterisation of denotational equality, arriving at a sound and complete calculus for affine relations?

\section{Extending GLA: Graphical Affine Algebra}

The answer to the first question is positive, and surprisingly simple. The syntax of Graphical Affine Algebra (GAA) extends (1) with just one additional connector

expressing the 'constant 1 ' behaviour. In other words, $\vdash$ can be regarded as a circuit component that emits a constant unit signal on its right interface: the intended relation is $\{(\bullet, 1)\}$. We will now sketch how this language extension allows us to capture the aforementioned examples.

Let us first consider $\mathbb{N}$-relations. Using $\vdash$, we define +

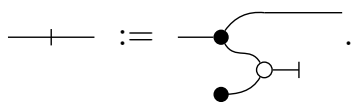

The result is a wire that can only carry 0 or 1 (i.e., any natural number $\leq 1)$. Given this, the mutual exclusion connector $(2)$ is simply the composition of 3 - with -1 .

Let us now switch to $\mathbb{R}$-relations. It is not difficult to see that the relation (3) is expressed by the following GAA diagram:

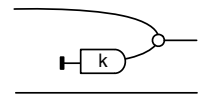

Indeed, as we shall see, adding the generator $\vdash$ is sufficient for expressing both $\mathbb{N}$ - and $\mathbb{R}$-affine relations - in fact, affine relations over any field $\mathbb{K}$.

As a final example, let us consider how to express the empty relation $\varnothing$-which is both $\mathbb{N}$ - and $\mathbb{R}$-affine-using our syntax.

$$
\mapsto \mathrm{O}
$$

Here the new $\vdash$ generator is composed with -0 . Operationally, this amounts to asserting " $1=0$ "; in terms of the relational interpretation, the result is the composition of $\{(\bullet, 1)\}$ with $\{(0, \bullet)\}$, which gives the empty relation.

\section{Equational Characterisations}

The answer to the second question is also positive. As our main technical contribution, we provide two sound and fully complete axiomatisations for GAA over affine relations, for the two semirings that are of interest in our applications: the case $R=\mathbb{N}$, and the case in which $R$ is a field $\mathbb{K}$.

The equational theories are simple, with only a few additional equations that govern the interaction of $\vdash$ with the remaining GLA primitives. A particularly interesting equation is shared by the two theories and concerns the properties of the empty relation (6). Recall that the composition of any relation with the empty relation, as well as the cartesian product of any relation with the empty relation, results in the empty relation. Equationally, $\vdash$ thus behaves analogously to logical false; in particular, we ought to be able to prove that

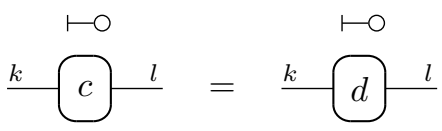

for any diagrams $c$ and $d$. We show that this can be accomplished with a single equation that "disconnects" a wire.

\section{E. Outline}

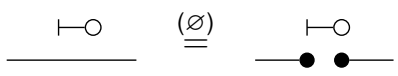

In Section II we introduce the syntax $A C_{i r c_{R}}$ of circuit diagrams - the basic language that we will use throughout the paper-with its semantics, in terms of relations of Rvectors. In Section III we restrict our focus to two domains of interpretation: the prop AffRel $\left.\right|_{\mathbb{K}}$ of affine relations over a field $\mathbb{K}$ and AffRel $_{\mathbb{N}}$ of affine relations over the natural numbers. In Section IV we introduce two corresponding equational theories $\mathrm{AIH}_{\mathbb{K}}$ and $\mathrm{ARC}$, and prove that they are fully complete for their intended semantics. The syntax, semantics, and equational theories involved are summarised in Figure 1. The next two sections are devoted to case studies that showcase the expressivity of our calculus. The first, in Section V, uses GAA over $\mathbb{N}$ to capture the calculus of stateless connectors, a coordination language for distributed systems [14] closely related to Reo [16] and BIP [17]. The second case study, in Section VI, uses GAA over $\mathbb{R}(x)$ to model the behaviour of electrical circuits, including not only passive components as [15], but also current and voltage sources. We prove by diagrammatic reasoning classic results about compositions of these components, and show how GAA allows us to detect undefined behaviour.

\section{The LANGUAGE OF CiRCUIT Diagrams}

\section{A. Syntax and Semantics}

We start with a simple language of circuit diagrams, given by the grammar below. Values $k$ in $-k$ - range over elements of a given semiring $\mathrm{R}$.

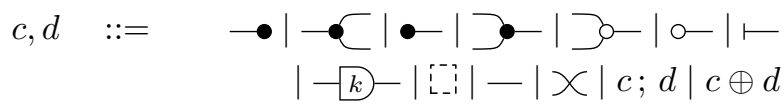

We write $A C i r c_{R}$ for the full language and $\operatorname{Circ}_{R}$ for the fragment without $\longmapsto$ (A stands for 'affine'). As mentioned 


\begin{tabular}{|c|c|c|c|c|}
\hline & {$[3],[7],[18]$} & [13] & \multicolumn{2}{|c|}{ This paper } \\
\hline Theory & \multicolumn{2}{|c|}{ Graphical Linear Algebra } & \multicolumn{2}{|c|}{ Graphical Affine Algebra } \\
\hline Syntax & $\operatorname{Circ}_{\mathbb{K}}$ & $\operatorname{Circ}_{\mathbb{N}}$ & ACirc $_{\mathbb{K}}$, Sec. II & ACirc $_{\mathbb{N}}$, Sec. II \\
\hline Semantics & $\operatorname{LinRel}_{\mathbb{K}}$ & AddRel & AffRel $_{\mathbb{K}}$, Sec. III & AffRel $_{\mathbb{N}}$, Sec. III \\
\hline Axioms & $\mathrm{IH}_{\mathbb{K}}$ & $\mathrm{RC}$ & $\mathrm{AlH}_{\mathbb{K}}$, Sec. IV & ARC, Sec. IV \\
\hline Embeds & $\begin{array}{l}\text { Signal Flow } \\
\text { Graphs }\end{array}$ & Petri Nets & $\begin{array}{c}\text { Electrical } \\
\text { circuits } \\
\text { Sec. VI }\end{array}$ & $\begin{array}{l}\text { Stateless } \\
\text { connectors } \\
\text { Sec. V }\end{array}$ \\
\hline
\end{tabular}

Fig. 1. Overview on GLA and GAA, in the notation of this paper.

in Section I, for different Rs, Circ $R$ is able to model linear dynamical systems [7], [19], phase-free quantum processes [5], Petri nets [13], and more. The focus of this paper is exploring the expressivity and the equational theories supported by the extended language $A \mathrm{Circ}_{\mathrm{R}}$.

Symbols of $A \operatorname{Circ}_{R}$ are rendered pictorially, as we will treat them formally as string diagrams [20] in due course (Section II-B). This also explains the use of two binary operations, sequential $(c ; d)$ and parallel $(c \oplus d)$ composition: they are those of monoidal categories.

The diagrammatic syntax is variable-free, but requires a simple sorting discipline. A sort is a pair $(k, l)$, with $k, l \in \mathbb{N}$; intuitively, $k$ and $l$ are the number of dangling wires on each side of a $\mathrm{ACirc}_{\mathrm{R}}$-diagram. We shall only consider terms that are sortable, according to the following rules.

$$
\begin{aligned}
& \longrightarrow:(1,0) \rightarrow(1,2) \quad \bigcirc-(2,1) \quad \circ:(0,1) \\
& \text { •- : }(0,1) \text { ○ : }(2,1) \vdash:(0,1)-k-:(1,1)
\end{aligned}
$$

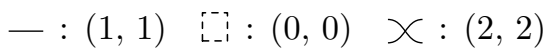

$$
\begin{aligned}
& \frac{c:\left(k_{1}, k_{2}\right) \quad d:\left(k_{2}, k_{3}\right)}{c ; d:\left(k_{1}, k_{3}\right)} \quad \frac{c:\left(k_{1}, l_{1}\right) \quad d:\left(k_{2}, l_{2}\right)}{c \oplus d:\left(k_{1}+k_{2}, l_{1}+l_{2}\right)}
\end{aligned}
$$

An easy induction confirms uniqueness of sorting: if $c:(k, l)$ and $c:\left(k^{\prime}, l^{\prime}\right)$, then $k=k^{\prime}$ and $l=l^{\prime}$.

The semantics $\langle\cdot\rangle_{R}$ of $A$ irc $_{R}$ is defined inductively by the clauses in Figure 2, where we write • for the unique R-vector of length zero.

Intuitively, -2 duplicates, - discards and $\bigcirc-$ sums values, whereas $\circ-$ produces zero values, $\vdash$ produces one value, and $-k-$ multiplies by $k$ a given value. The mirror images $7-, \rightarrow$ have behaviour defined symmetrically with respect to $\prec$ and $\bullet$. Finally, behaviours combine sequentially, where values synchronise along the common boundary of diagrams, or in parallel, where values are simply stacked. Note that $\langle\cdot\rangle_{R}$ is defined in terms of relations rather than functions; thus it is neutral with respect to flow directionality. For further discussion on this point, see [8].

\section{B. From Terms to String Diagrams}

Our goal is to characterise semantic equivalence in $\mathrm{ACirc}_{R}$ equationally, for different choices of R. In each case, these equations contain the laws of symmetric monoidal categories (SMCs). Thus it makes sense to move from raw terms, as in (7), to string diagrams: this is the remit of the subsection.

First, we enhance our graphical notation by depicting $c:(k, l)$ as ${ }^{k}-c ; d$ as $c^{k_{1}} c^{k_{2}} d{ }^{k_{3}}$ and $c \oplus d$ as $\frac{k_{2}}{d l^{l_{2}}}$, where the labelled wire $\frac{k}{-}$ stands for a stack of $k$ wires. We often omit wire labels when it does not lead to confusion.

The laws of SMCs are given in Figure (3) in the graphical notation. They yields a structural equivalence on $A C_{i r C_{R}}$-terms, which is preserved by the semantics. More precisely, writing $\equiv$ for the smallest congruence over ACirc $C_{R}$-terms generated by the equations in Figure 3, we have that $c \equiv d$ implies $\langle c\rangle_{R}=\langle d\rangle_{R}$. Because of this observation, henceforth we shall consider the terms of $\mathrm{ACirC}_{\mathrm{R}}$ as arrows of an SMC, which by a mild abuse of notation we will also denote $A C$ irc $C_{R}$. In fact, $\mathrm{ACirC}_{\mathrm{R}}$ is a specific kind of SMC, known as a prop.

Definition 1. A prop is a symmetric strict monoidal category with objects the natural numbers, with $k \oplus l$ defined by the addition $k+l$. A prop morphism $\mathcal{F}: \mathrm{C} \rightarrow \mathrm{D}$ is a symmetric monoidal functor from $\mathrm{C}$ to $\mathrm{D}$ that is identity on objects.

ACirc $_{\mathrm{R}}$ is defined as a prop with arrows $k \rightarrow l$ sorted terms $c:(k, l)$ of the corresponding syntax modulo $\equiv$, with sequential composition $c ; d$, monoidal product $c \oplus d$, and symmetries defined by the corresponding operations in (7) (c.f. [19, Definition 2.3] for the details of the free construction of a prop from a syntax).

For uniformity, we shall also model the semantic domains of our calculus as props. This is based on the definition below.

Definition $2\left(\operatorname{Re}_{\mathrm{R}}\right)$. Given a semiring $\mathrm{R}$, let $\mathrm{Re}_{\mathrm{R}}$ be the prop with arrows $k \rightarrow l$ relations $R$ from $X^{k}$ to $X^{l}$, i.e. $R \subseteq X^{k} \times$ $X^{l}$. Given $R: k_{1} \rightarrow k_{2}$ and $S: k_{2} \rightarrow k_{3}$, their composition $R ; S: k_{1} \rightarrow k_{3}$ is

$$
\begin{array}{r}
\left\{(\boldsymbol{x}, \boldsymbol{z}): \boldsymbol{x} \in X^{k_{1}}, \boldsymbol{z} \in X^{k_{3}} \text { and there exists } \boldsymbol{z} \in X^{k_{2}}\right. \\
\text { such that }(\boldsymbol{x}, \boldsymbol{y}) \in R \text { and }(\boldsymbol{y}, \boldsymbol{z}) \in S\} .
\end{array}
$$

Given $R: k_{1} \rightarrow l_{1}$ and $S: k_{2} \rightarrow l_{2}$ their monoidal product is obtained by taking their cartesian product, i.e. $R \oplus S$ : $k_{1}+k_{2} \rightarrow l_{1}+l_{2}$ is the relation

$$
\begin{array}{r}
\left\{\left(\left(\begin{array}{l}
\boldsymbol{x}_{\mathbf{1}} \\
\boldsymbol{x}_{\mathbf{2}}
\end{array}\right),\left(\begin{array}{l}
\boldsymbol{y}_{\mathbf{1}} \\
\boldsymbol{y}_{\mathbf{2}}
\end{array}\right)\right): \boldsymbol{x}_{\mathbf{1}} \in X^{k_{1}}, \boldsymbol{x}_{\mathbf{2}} \in X^{k_{2}}, \boldsymbol{y}_{\mathbf{1}} \in X^{l_{1}},\right. \\
\left.\boldsymbol{y}_{\mathbf{2}} \in X^{l_{2}} \text { such that }\left(\boldsymbol{x}_{\mathbf{1}}, \boldsymbol{y}_{\mathbf{1}}\right) \in R \text { and }\left(\boldsymbol{x}_{\mathbf{2}}, \boldsymbol{y}_{\mathbf{2}}\right) \in S\right\} .
\end{array}
$$

Identities and symmetries are defined in the obvious way.

Note that our inductive definition of Figure 2 yields a morphism of props $\langle-\rangle_{R}:$ ACirc $_{R} \rightarrow$ Rel $_{R}$. Functoriality here means that the interpretation is compositional with respect to the operations ; and $\oplus$.

\section{Compact closed structure}

String diagrams $\bigcirc \bullet$, called cup and $\bullet \bullet$, cap, play a special role. Their behaviour, according to the semantics $\langle\cdot\rangle_{R}$, 


$$
\begin{aligned}
& \langle\longrightarrow\rangle_{\mathrm{R}}:=\{(x, \bullet) \mid x \in \mathrm{R}\} \quad\langle\longrightarrow\rangle_{\mathrm{R}}:=\left\{\left(x,\left(\begin{array}{l}
x \\
x
\end{array}\right)\right) \mid x \in \mathrm{R}\right\} \quad\langle\bullet\rangle_{\mathrm{R}}:=\{(\bullet, x) \mid x \in \mathrm{R}\} \quad\left\langle\supset_{-}\right\rangle_{\mathrm{R}}:=\left\{\left(\left(\begin{array}{l}
x \\
x
\end{array}\right), x\right) \mid x \in \mathrm{R}\right\} \\
& \langle\mathcal{O}-\rangle_{\mathrm{R}}:=\left\{\left(\left(\begin{array}{l}
x \\
y
\end{array}\right), x+y\right) \mid x, y \in \mathrm{R}\right\} \quad\langle\mathrm{O}-\rangle_{\mathrm{R}}:=\{(\bullet, 0)\} \quad\langle-k-\rangle_{\mathrm{R}}:=\{(x, k \cdot x) \mid x \in \mathrm{R}\} \quad\langle\vdash\rangle_{\mathrm{R}}:=\{(\bullet, 1)\} \\
& \left\langle\begin{array}{c}
i \\
i \\
L \\
i
\end{array}\right\rangle_{\mathrm{R}}:=\{(\bullet, \bullet)\} \quad\langle-\rangle_{\mathrm{R}}:=\{(x, x) \mid x \in \mathrm{R}\} \quad\langle X\rangle_{\mathrm{R}}:=\left\{\left(\left(\begin{array}{l}
x \\
y
\end{array}\right),\left(\begin{array}{l}
y \\
x
\end{array}\right)\right) \mid x, y \in \mathrm{R}\right\} \\
& \langle c ; d\rangle_{\mathrm{R}}:=\left\{(\boldsymbol{a}, \boldsymbol{b}) \mid \exists \boldsymbol{w} \cdot(\boldsymbol{a}, \boldsymbol{w}) \in\langle c\rangle_{\mathrm{R}},(\boldsymbol{w}, \boldsymbol{b}) \in\langle d\rangle_{\mathrm{R}}\right\} \quad\langle c \oplus d\rangle_{\mathrm{R}}:=\left\{\left(\left(\begin{array}{c}
\boldsymbol{a}_{\mathbf{1}} \\
\boldsymbol{a}_{\mathbf{2}}
\end{array}\right),\left(\begin{array}{c}
\boldsymbol{b}_{\mathbf{1}} \\
\boldsymbol{b}_{\mathbf{2}}
\end{array}\right)\right) \mid\left(\boldsymbol{a}_{\mathbf{1}}, \boldsymbol{a}_{\mathbf{2}}\right) \in\langle c\rangle_{\mathrm{R}},\left(\boldsymbol{b}_{\mathbf{1}}, \boldsymbol{b}_{\mathbf{2}}\right) \in\langle d\rangle_{\mathrm{R}}\right\}
\end{aligned}
$$

Fig. 2. Semantics of $A \operatorname{Circ}_{R}$.

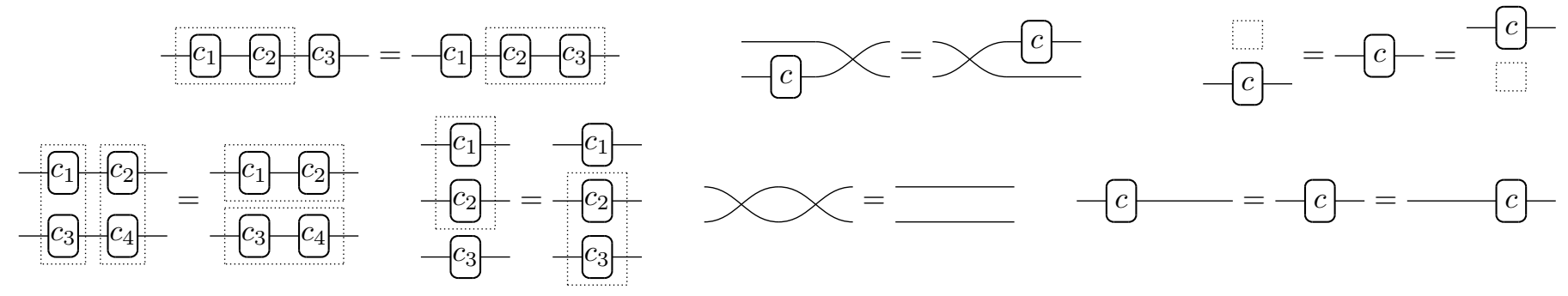

Fig. 3. Laws of Symmetric Monoidal Categories. Sort labels are omitted for readability.

intuitively forces the two ports on the left (respectively right) to carry the same resources, thus acting as left (right) feedback:

$$
\langle\supset \bullet\rangle_{\mathrm{R}}=\left\{\left(\left(\begin{array}{l}
x \\
x
\end{array}\right), \bullet\right) \mid x \in X\right\}\langle\bullet \bullet\rangle_{\mathrm{R}}=\left\{\left(\bullet,\left(\begin{array}{l}
x \\
x
\end{array}\right)\right) \mid x \in X\right\}
$$

Using these diagrams (along with - and $\chi$ ) as building blocks, is it possible to define for each $k \in \mathbb{N}$, diagrams $k \bullet \bullet: k+k \rightarrow 0$ and $\bullet \bullet k$ : $0 \rightarrow k+k$ with semantics

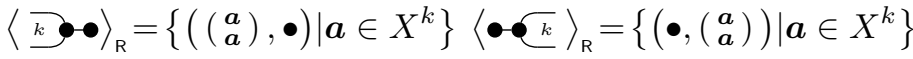

See e.g. $[19, \S 5.1]$ for details. These arrows give rise to a (self-dual) compact closed structure [21], that is, they satisfy

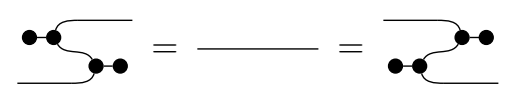

As for identities and symmetries, we will often omit the label $k$ on cups and caps for readability. The graphical language of compact closed props allows us to bend wires at will, treating them as unoriented edges between the connection points of individual components. It also allows the introduction of "right-to-left" versions of each generator in our diagrammatic syntax. We explicitly introduce these counterparts as syntactic sugar, since they will be used in subsequent sections.

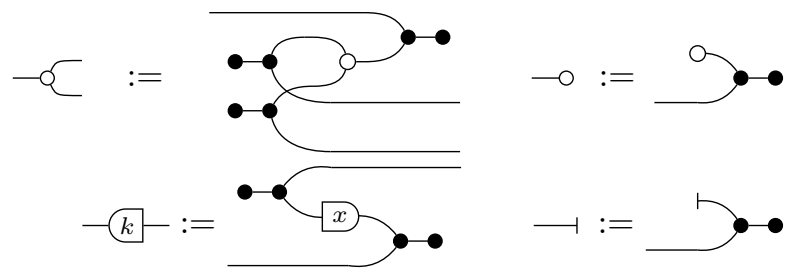

The associated behaviour, in each case, is the opposite relation: for example, $\langle-\mathcal{C}\rangle_{\mathrm{R}}=\left\{\left(n+m,\left(\begin{array}{c}n \\ m\end{array}\right)\right) \mid n, m \in \mathbb{N}\right\}$.

\section{Examples}

We conclude this section by showcasing the expressivity of $A C_{i r c_{R}}$. The first two examples are captured by the fragment $\operatorname{Circ}_{R}$, whereas the last two are genuinely affine (they make use of $\vdash$ ), and anticipate the case studies of Section V and VI.

a) Lowpass filter: Take $\mathrm{R}$ to be $\mathbb{R}(x)$. The idea is that elements of $\mathbb{R}(x)$ express Laplace-transformed signals in the frequency domain. In other words, multiplication by $x$ corresponds to differentiation w.r.t. the time variable. The $\operatorname{Circ}_{\mathbb{R}(x)}$-diagram on the left below denotes a low-pass filter: a filter that attenuates signals with frequencies higher than a certain cutoff $\left(\leq \frac{1}{2 \pi \tau}\right)$ and lets through those that are lower. The semantics of the diagram, on the right below, allows us to retrieve the transfer function (the ratio of the left and right boundary signals) of the filter.

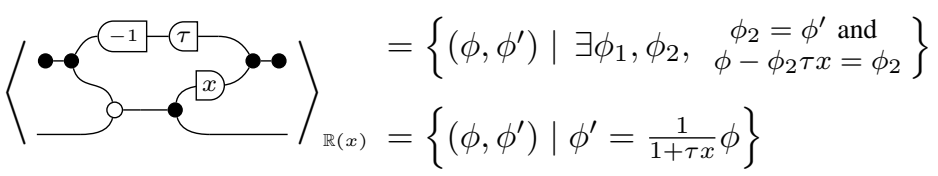

b) Less than or equal to: Again for $\mathrm{R}=\mathbb{N}$, consider the diagram .0 - of $\operatorname{Circ}_{\mathbb{N}}$. One can check that

$$
\begin{aligned}
\langle\mathcal{O} & =\left\{(m, n) \mid \exists m^{\prime} \cdot m+m^{\prime}=n\right\} \\
& =\{(m, n) \mid m \leq n\}
\end{aligned}
$$

Observe that if, instead of $\mathbb{N}$, we parametrise the theory over a semiring with additive inverses, such as $\mathbb{R}$, then the same diagram collapses to the total relation $\langle\longrightarrow \bullet\rangle_{\mathbb{R}}=\mathbb{R} \times \mathbb{R}$.

c) Mutual exclusion: For the diagram $\mathrm{O}+$ of $\operatorname{Circ}_{\mathbb{N}}$ from (4), first notice that $\langle-\rangle_{\mathrm{N}}$ is by definition equal to

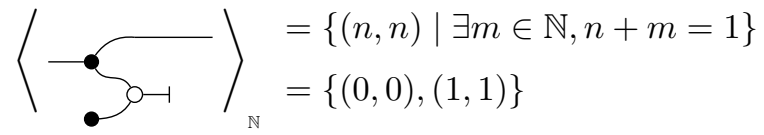


Then we can derive that $\left(\left(\begin{array}{c}m \\ n\end{array}\right), z\right) \in\langle\supset+\rangle_{\mathbb{N}}$ whenever $z=m+n \leq 1$. We have, therefore

$$
\langle\supset-\rangle_{\mathbb{N}}=\left\{\left(\left(\begin{array}{l}
1 \\
0
\end{array}\right), 1\right),\left(\left(\begin{array}{l}
0 \\
1
\end{array}\right), 1\right),\left(\left(\begin{array}{l}
0 \\
0
\end{array}\right), 0\right)\right\},
$$

that is, at most one of the upper and lower port on the left may synchronise with the port on the right. This diagram thus implements mutual exclusion.

d) Ideal voltage source: The behaviour of electrical components is expressed as a voltage-current relationship between their terminals. As was done in [15] we will use one wire to denote voltage and another to denote the current, each modelled as a real number. With this interpretation, a simple voltage source with fixed voltage $k$ is denoted by the same ACirc $_{\mathbb{R}}$ diagram as in (5) and we can see that its denotation coincides with (3):

$$
\langle\underset{\mathrm{k}}{\mathrm{\textrm {N }}}\rangle_{\mathbb{R}}=\left\{\left(\left(\begin{array}{c}
\phi_{1} \\
i
\end{array}\right),\left(\begin{array}{c}
\phi_{2} \\
i
\end{array}\right)\right) \mid \phi_{1}+k=\phi_{2}\right\}
$$

\section{CATEgories OF AFFine RElations}

In the remainder of the paper we focus on two instances of $R$, motivated by applications: the case when $R$ is a field $\mathbb{K}$, and the case when $R$ is the semiring $\mathbb{N}$ of natural numbers.

Our overall goal is to equip $A \operatorname{Circ}_{\mathbb{K}}$ and $A C i r c_{\mathbb{N}}$ with sound and fully complete axiomatisations with respect to suitable classes of relations in the semantic domain. This is articulated in two steps. In this section, we identify these classes as $\mathbb{K}$-affine relations and $\mathbb{N}$-affine relations, showing that they form props $A f f R e l_{\mathbb{K}}$ and $A f f R e l_{\mathbb{N}}$ respectively. The next section will conclude the characterisation by introducing equational theories for $A C_{i r c}$ and $A C i r c_{\mathbb{N}}$ which are sound and fully complete with respect to $A f f R e I_{\mathbb{K}}$ and $A f f R e l_{\mathbb{N}}$.

\section{A. Affine relations over a field}

Fix a field $\mathbb{K}$. We recall the concept of linear relation.

Definition 3 ( [3], [7]). A $\mathbb{K}$-linear relation of type $k \rightarrow l$ is a linear subspace of $\mathbb{K}^{k} \times \mathbb{K}^{l}$, considered as a $\mathbb{K}$-vector space. $\mathbb{K}$-linear relations form a sub-prop $\operatorname{LinRe}_{\mathbb{K}}$ of $\operatorname{Rel}_{\mathbb{K}}$, with composition defined by (7)-(8).

By analogy, we define affine relations from affine subspaces.

Definition 4. An affine subspace of $\mathbb{K}^{d}$ is a subset $V \subseteq \mathbb{K}^{d}$ that is either empty or for which there exists a vector $\mathbf{a} \in \mathbb{K}^{d}$ and a linear subspace $L$ of $\mathbb{K}^{d}$ such that $V=\boldsymbol{a}+L:=$ $\{\boldsymbol{a}+\boldsymbol{x} \mid \boldsymbol{x} \in L\}$.

$A \mathbb{K}$-affine relation of type $k \rightarrow l$ is an affine subspace of $\mathbb{K}^{k} \times \mathbb{K}^{l}$, considered as a $\mathbb{K}$-vector space.

Our next goal is to show that $\mathbb{K}$-affine relations form a prop. To this aim, it is useful to give a construction that allows us to relate linear and affine relations systematically, using homogenisation, a well-known technique of affine and convex geometry. To every affine relation we associate a linear relation in a unique way, by embedding it into a space with an additional dimension. First, for a set $X \subseteq \mathbb{K}^{k} \times \mathbb{K}^{l}$, let

$$
{ }^{0} X=\left\{\left(\left(\begin{array}{c}
0 \\
\boldsymbol{a}
\end{array}\right), \boldsymbol{b}\right) \mid(\boldsymbol{a}, \boldsymbol{b}) \in X\right\} \subseteq \mathbb{K}^{k+1} \times \mathbb{K}^{l}
$$

Definition 5. Let $R: k \rightarrow l$ be an affine relation given by $(\boldsymbol{a}, \boldsymbol{b})+L$. Its homogenisation is the linear relation $\hat{R}: k+1 \rightarrow$ $l$ defined as $\hat{R}=\left(\left(\begin{array}{c}1 \\ \boldsymbol{a}\end{array}\right), \boldsymbol{b}\right)+{ }^{0} L$.

Homogenisation satisfies an useful property:

$$
(\boldsymbol{a}, \boldsymbol{b}) \in R \quad \text { if and only if } \quad\left(\left(\begin{array}{l}
1 \\
\boldsymbol{a}
\end{array}\right), \boldsymbol{b}\right) \in \widehat{R} .
$$

We will now use homogenisation to prove affine relations close under relational composition. If $R: k \rightarrow l$ and $S$ : $l \rightarrow p$ are affine relations, we cannot directly compose their homogenisations $\widehat{R}: k+1 \rightarrow l$ and $\widehat{S}: l+1 \rightarrow p$ as linear relations, as the types do not match.

We can, however, interpret the homogenisation construction as embedding affine relations into a subcategory of the coKleisli category of the $(-)+1$ comonad, with structural natural transformations $\mu:(-)+1 \rightarrow(-)+2$ and $\epsilon:(-)+1 \rightarrow$ id:

$$
\mu_{k}=\langle\underset{k}{\longrightarrow}\rangle_{\mathbb{K}} \text { and } \epsilon_{k}=\left\langle\frac{\longrightarrow}{k}\right\rangle_{\mathbb{K}}
$$

The comonad laws are a consequence of the comonoid law for $-\mathcal{C}$ and are easy to check. For $U: k+1 \rightarrow l$ and $V: l+1 \rightarrow p$ two linear relations, their coKleisli composition $U \hat{;} V$ is:

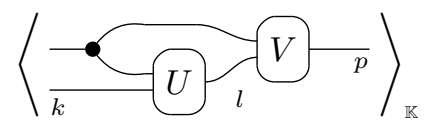

We are now able to prove the following closure property.

Proposition 6. The composite (7) of two $\mathbb{K}$-affine relations is a $\mathbb{K}$-affine relation.

Proof. Let $R: k \rightarrow l$ and $S: l \rightarrow p$ be two $\mathbb{K}$-affine relations. If $(\boldsymbol{x}, \boldsymbol{z}) \in R ; S$ there exists $\boldsymbol{y} \in \mathbb{K}^{k}$ such that $(\boldsymbol{x}, \boldsymbol{y}) \in R$ and $(\boldsymbol{y}, \boldsymbol{z}) \in S$. So $\left(\left(\begin{array}{l}1 \\ \boldsymbol{x}\end{array}\right), \boldsymbol{y}\right) \in \widehat{R}$ and $\left(\left(\begin{array}{l}1 \\ \boldsymbol{y}\end{array}\right), \boldsymbol{z}\right) \in \widehat{S}$ and therefore $\left(\left(\begin{array}{c}1 \\ \boldsymbol{x}\end{array}\right), \boldsymbol{z}\right) \in \widehat{R} \widehat{;} \widehat{S}$. As long as $R ; S$ is nonempty (in which case we are done), we can always find a basis of $\widehat{R} \widehat{;} \widehat{S}$ of the form $\left\{\left(\left(\begin{array}{c}1 \\ \boldsymbol{e}\end{array}\right), \boldsymbol{f}\right),\left(\left(\begin{array}{c}0 \\ \boldsymbol{e}_{1}\end{array}\right), \boldsymbol{f}_{1}\right), \ldots,\left(\left(\begin{array}{c}0 \\ \boldsymbol{e}_{n}\end{array}\right), \boldsymbol{f}_{n}\right)\right\}$. Then, clearly $R ; S=(\boldsymbol{e}, \boldsymbol{f})+L$ where $L$ is the linear subspace spanned by $\left\{\left(\boldsymbol{e}_{1}, \boldsymbol{f}_{1}\right), \ldots,\left(\boldsymbol{e}_{n}, \boldsymbol{f}_{n}\right)\right\}$.

As a result, we can define the prop $\left.A f f R e\right|_{\mathbb{K}}$ of $\mathbb{K}$-affine relations, as a sub-prop of $\operatorname{Rel}_{\mathbb{K}}$.

\section{B. Affine relations over the semiring $\mathbb{N}$}

When the semiring is $\mathbb{N}$, the absence of additive and multiplicative inverses yields a different semantics, which requires slightly more work than the $\mathbb{K}$-affine case. Our departure point is the notion of additive relation.

Definition 7 ( [13]). An additive relation of type $k \rightarrow l$ is a subset $R \subseteq \mathbb{N}^{k} \times \mathbb{N}^{l}$ such that $(i)(\mathbf{0}, \mathbf{0}) \in R$ and (ii) if $(\boldsymbol{a}, \boldsymbol{b}),\left(\boldsymbol{a}^{\prime}, \boldsymbol{b}^{\prime}\right) \in R$ then $\left(\boldsymbol{a}+\boldsymbol{a}^{\prime}, \boldsymbol{b}+\boldsymbol{b}^{\prime}\right) \in R$.

Every pair $(\boldsymbol{a}, \boldsymbol{b}) \in \mathbb{N}^{k} \times \mathbb{N}^{l}$ generates an additive relation $\langle(\boldsymbol{a}, \boldsymbol{b})\rangle=\{(n \boldsymbol{a}, n \boldsymbol{b}) \mid n \in \mathbb{N}\}$. More generally, for a finite set $G=\left\{\left(\boldsymbol{a}_{1}, \boldsymbol{b}_{1}\right), \cdots,\left(\boldsymbol{a}_{p}, \boldsymbol{b}_{p}\right)\right\}$ of points in $\mathbb{N}^{k} \times \mathbb{N}^{l}$, we write $\langle G\rangle$ for the additive relation

$$
\langle G\rangle=\left\{\sum_{i=1}^{p} n_{i}\left(\boldsymbol{a}_{i}, \boldsymbol{b}_{i}\right) \mid n_{1}, \ldots, n_{p} \in \mathbb{N}\right\} .
$$


For our characterisation only finitely generated additive relations are relevant.

Definition 8. An additive relation $R: k \rightarrow l$ is finitely generated (f.g.) if there exists a finite set of vectors $G=$ $\left\{\left(\boldsymbol{a}_{1}, \boldsymbol{b}_{1}\right), \cdots,\left(\boldsymbol{a}_{p}, \boldsymbol{b}_{p}\right)\right\}$ such that $R=\langle G\rangle$. F.g. $\mathbb{N}$-additive relations form a sub-prop AddRel of $\operatorname{Rel}_{\mathbb{N}}$.

Example 9. The two pictures in the left of Figure 4 represents the additive relations of type $1 \rightarrow 1$ generated by $\{(1,2),(3,1)\}$ and $\{(1,3),(2,2),(4,1)\}$ respectively.

Henceforward, when we say "additive relation", we mean $f$. . additive relation. Differently from the linear case, closure under composition of additive relations is a nontrivial fact [13]. The following is another important difference between the linear and additive worlds.

Proposition 10 ( [13, Proposition 23]). Every additive relation has a unique minimal (for inclusion) generating set, called its Hilbert basis.

We now move to defining $\mathbb{N}$-affine relations. Recall that the Minkowski sum of sets $C, D \subseteq \mathbb{N}^{d}$ is $C+D=$ $\{\boldsymbol{c}+\boldsymbol{d} \mid \boldsymbol{c} \in C, \boldsymbol{d} \in D\}$. If one of these is a singleton, e.g. $C=\{c\}$, we will abuse notation and write $c+D$. Note that $\varnothing+D=\varnothing$.

Definition 11. $A \mathbb{N}$-affine relation $R: k \rightarrow l$ is a set $R \subseteq$ $\mathbb{N}^{k} \times \mathbb{N}^{l}$ for which there exists finite $B, D \subseteq \mathbb{N}^{k} \times \mathbb{N}^{l}$ such that $R=\bigcup_{(\boldsymbol{a}, \boldsymbol{b}) \in B}\{(\boldsymbol{a}, \boldsymbol{b})+\langle D\rangle\}$. Elements of $B$ are called base points and those of $D$ directions.

The first noticeable difference with affine relations over a field is that $\mathbb{N}$-affine relations can have more than one base point. If $B=\{(\boldsymbol{a}, \boldsymbol{b})\}$ is a singleton, $R=(\boldsymbol{a}, \boldsymbol{b})+D$ is the translation of an additive relation by $(\boldsymbol{a}, \boldsymbol{b})$. If $B=\{(\mathbf{0}, \mathbf{0})\}$, $R$ is an additive relation. Thus every additive relation $R$ is $\mathbb{N}$ affine: take $B=\{(\mathbf{0}, \mathbf{0})\}$ and $D$ to be a generating set of $R$. An $\mathbb{N}$-affine relation is, therefore, a finite union of translated additive relations.

Example 12. The two pictures on the right of Figure 4 represent the two $\mathbb{N}$-affine relations of type $1 \rightarrow 1$ with respective bases $\{(2,2),(4,2)\}$ and $\{(1,2)\}$, and respective directions $\{(1,2),(3,1)\}$ and $\{(1,3),(2,2),(4,1)\}$.

Note that every finite subset $S$ of $\mathbb{N}^{k+l}$ is also $\mathbb{N}$-affine (by setting $B=S$ and $D=\{(\mathbf{0}, \mathbf{0})\}$ ). Finally, $\varnothing$ is $\mathbb{N}$-affine (e.g. by taking $B=\varnothing$ ) but not additive.

As for $\mathbb{K}$-affine relations, we can define the homogenisation of an $\mathbb{N}$-affine relation. For this we will also need the following notation, for $X \subseteq \mathbb{N}^{k} \times \mathbb{N}^{l}$ :

$$
{ }^{1} X=\left\{\left(\left(\begin{array}{c}
1 \\
\boldsymbol{a}
\end{array}\right), \boldsymbol{b}\right) \mid(\boldsymbol{a}, \boldsymbol{b}) \in X\right\} \subseteq \mathbb{N}^{k+1} \times \mathbb{N}^{l} .
$$

Definition 13. Let $R: k \rightarrow l$ be an $\mathbb{N}$-affine relation with base points $B$ and directions $D$. Its homogenisation is the additive relation $\widehat{R}: k+1 \rightarrow l$ defined as $\widehat{R}=\left\langle{ }^{1} B \cup{ }^{0} D\right\rangle$.
As for $\mathbb{K}$-affine relations, the homogenisation of $\mathbb{N}$-affine relations satisfies the crucial property of equation (11). We can now prove that $\mathbb{N}$-affine relations form a category.

Proposition 14. The composite (7) of two $\mathbb{N}$-affine relations is an $\mathbb{N}$-affine relation.

Proof. Let $R: k \rightarrow l$ and $S: l \rightarrow p$ be $\mathbb{N}$-affine relations and $R ; S$ their composite. We can obtain the base points and directions of $R ; S$ from the Hilbert basis $H$ of the additive relation $\widehat{R} \widehat{;} \widehat{S}$. If $(\boldsymbol{a}, \boldsymbol{c}) \in R ; S$, there exists $\boldsymbol{b} \in \mathbb{N}^{k}$ such that $(\boldsymbol{a}, \boldsymbol{b}) \in R$ and $(\boldsymbol{b}, \boldsymbol{c}) \in S$. So $\left(\left(\begin{array}{l}1 \\ \boldsymbol{a}\end{array}\right), \boldsymbol{b}\right) \in \widehat{R}$ and $\left(\left(\begin{array}{l}1 \\ \boldsymbol{b}\end{array}\right), \boldsymbol{c}\right) \in \widehat{S}$ and therefore $\left(\left(\begin{array}{l}1 \\ \boldsymbol{a}\end{array}\right), \boldsymbol{c}\right) \in$ $\widehat{R} \widehat{;} \widehat{S}$. We can decompose this last pair into a weighted sum of $H$-elements $\left(\left(\begin{array}{c}1 \\ \boldsymbol{a}\end{array}\right), \boldsymbol{c}\right)=\left(\left(\begin{array}{c}1 \\ \boldsymbol{f}\end{array}\right), \boldsymbol{g}\right)+\sum_{i=1}^{m} n_{i}\left(\left(\begin{array}{c}0 \\ \boldsymbol{d}_{i}\end{array}\right), \boldsymbol{e}_{i}\right)$, where $\left(\left(\begin{array}{c}1 \\ \boldsymbol{f}\end{array}\right), \boldsymbol{g}\right),\left(\left(\begin{array}{c}0 \\ \boldsymbol{d}_{i}\end{array}\right), \boldsymbol{e}_{i}\right) \in H$ and $n_{i} \in \mathbb{N}$ for $1 \leq$ $i \leq m$. Then, $(\boldsymbol{a}, \boldsymbol{c})=(\boldsymbol{f}, \boldsymbol{g})+\sum_{i=1}^{m} n_{i}\left(\boldsymbol{d}_{i}, \boldsymbol{e}_{i}\right)$. Therefore, since $(\boldsymbol{a}, \boldsymbol{c})$ was arbitrary, we conclude that $R ; S=$ $\bigcup_{(\boldsymbol{x}, \boldsymbol{y}) \in B}\{(\boldsymbol{x}, \boldsymbol{y})+\langle D\rangle\}$, with $B=\left\{(\boldsymbol{f}, \boldsymbol{g}) \mid\left(\left(\begin{array}{l}1 \\ \boldsymbol{f}\end{array}\right), \boldsymbol{g}\right) \in H\right\}$ and $D=\left\{(\boldsymbol{d}, \boldsymbol{e}) \mid\left(\left(\begin{array}{l}0 \\ \boldsymbol{d}\end{array}\right), \boldsymbol{e}\right) \in H\right\}$.

As a result, we can define the prop $A_{f f R e} \mathbb{N}_{\mathbb{N}}$ of $\mathbb{N}$-affine relations, as a sub-prop of $\operatorname{Rel}_{\mathbb{N}}$.

\section{Axiomatising AfFine Relations}

This section contains the main technical contribution of the paper, namely the introduction of equational theories for $A_{C i r c}$ and $A C i r c_{\mathbb{N}}$ that are sound and fully complete for their affine relation semantics. In each of the two cases, we first introduce the equational theory and its properties. Then we show that there is a prop isomorphism between the syntax modulo the equations, and affine relations. The full completeness result follows from the isomorphism.

\section{A. Axiomatising $\mathbb{K}$-affine relations}

As in the previous section, we start with the (non-affine) linear case. It is proven in [3], [7] that the quotient of Circ by the equations of interacting Hopf algebras (reported in Fig. 6 , Appendix A), which we call $I_{\mathbb{K}}$, is isomorphic to LinRel $_{\mathbb{K}}$.

Our characterisation will extend this isomorphism to affine systems. The challenge is to identify equations that govern the behaviour of the new generator $\vdash$. We claim that adding the following three equations is sufficient:

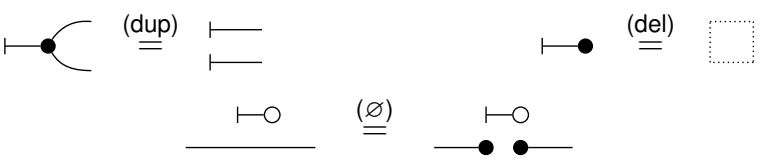

First, let us explain the new axioms. The first two say that $\vdash \quad$ can be deleted and copied by the comonoid structure, just like ${ }^{-}-$. This has the effect of constraining the interpretation of $\vdash \quad$ as a 0 -ary functional relation, i.e. it is a constant.

More interestingly, the third equation is justified by the possibility of expressing the empty set, by, for example,

$$
\langle\vdash \circ\rangle_{\mathbb{K}}=\{(\bullet, 1)\} ;\{(0, \bullet)\}=\varnothing .
$$

As mentioned previously, $\varnothing$ is an affine relation that is not linear. Since for any $R$ and $S$ in $\operatorname{Rel}_{\mathrm{R}}, \varnothing \oplus R=\varnothing \oplus S=$ 

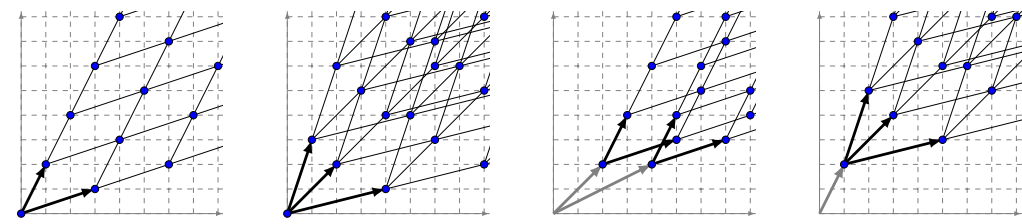

Fig. 4. Two additive and two $\mathbb{N}$-affine relations.

$\varnothing$, composing or taking the monoidal product of $\varnothing$ with any relation results in $\varnothing$. Thus $\varnothing$ is analogous to logical false.

Definition 15. The prop $\mathrm{AlH}_{\mathbb{K}}$ (affine interacting Hopf algebras) is the quotient of $\mathrm{ACirc}_{\mathbb{K}}$ by the equations of $\mathrm{IH}_{\mathbb{K}}$ (Fig. 6, Appendix A) plus (dup), (del) and ( $\varnothing)$.

We are going to show that $\mathrm{AlH}_{\mathbb{K}}$ is isomorphic to AffRel $\left.\right|_{\mathbb{K}}$. First, the following lemma formalises the preceding discussion about equation $(\varnothing)$.

Lemma 16. For any two arrows $c, d: k \rightarrow l$ of $\mathrm{AlH}_{\mathbb{K}}$,

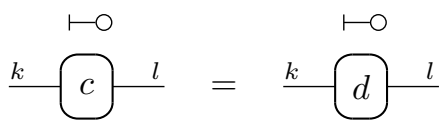

Proof. In Appendix C-A.

We are now ready to prove our characterisation result. Because all the equations of $A H_{\mathbb{K}}$ are sound in LinRel $\mathbb{K}_{\mathbb{K}}$, we can define a prop morphism $\llbracket-\rrbracket_{\mathbb{K}}: \mathrm{AlH}_{\mathbb{K}} \rightarrow \operatorname{LinRe}_{\mathbb{K}}$ inductively by the same clauses (Figure 2 ) of $\langle\cdot\rangle_{\mathbb{K}}$.

Theorem 17. $\llbracket \cdot \rrbracket_{\mathbb{K}}: \mathrm{AlH}_{\mathbb{K}} \rightarrow \operatorname{LinRe}_{\mathbb{K}}$ is a prop isomorphism.

Proof. First, we show that $\llbracket \cdot \rrbracket_{\mathrm{K}}$ is full. Diagrammatically, homogenisation means that $\mathbb{K}$-affine relations can be thought of as $\mathbb{K}$-linear relations with an extra dangling wire for the additional dimension. Because the restriction of $\llbracket-\rrbracket_{\mathbb{K}}$ to a functor $\mathrm{IH}_{\mathbb{K}} \rightarrow$ LinRel $_{\mathbb{K}}$ is well-defined and an isomorphism (thus, also full) [3, Theorem 6.4], we can always obtain a string diagram in $\mathrm{IH}_{\mathbb{K}}$ for the homogenisation $\hat{R}$ of an affine relation $R$. Then, we can use generator $\vdash$ to plug this wire, obtaining a string diagram

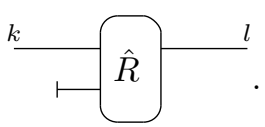

Finally, equation (11) implies that

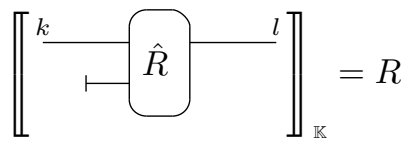

proving that $\llbracket-\rrbracket_{\mathbb{K}}$ is full. It remains to show that $\llbracket-\rrbracket_{K}$ is faithful. We will use a normal form argument, which relies the isomorphism of $\mathrm{IH}_{\mathbb{K}}$ and LinRe $\left.\right|_{\mathbb{K}}$ [3, Theorem 6.4]. Let $d: k \rightarrow l$ be a diagram in $\mathrm{AlH}_{\mathbb{K}}$. By naturality of the symmetry we may write $d$ as follows:

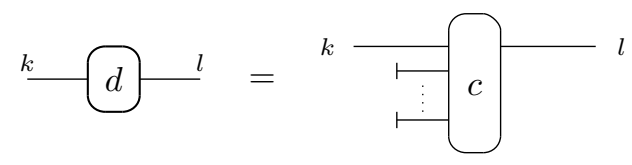

for some diagram $c$, in the image of the embedding $\mathrm{IH}_{\mathbb{K}} \hookrightarrow$ $\mathrm{AlH}_{\mathbb{K}}$. In graphical terms, we have pulled all copies of $\vdash$ up and down, past the rest of the diagram which represents some linear relation $c$. We may now simplify (17):

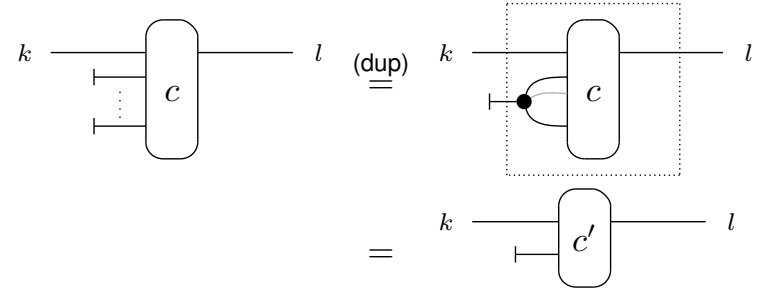

where $c^{\prime}$ is the diagram enclosed in the dotted box. Finally, from the span normal form for linear relations [3, Theorem 6.2], we can find a diagram $e: p \rightarrow l+1+k$ representing some matrix $\mathcal{M}_{e}$ (i.e. $\llbracket e \rrbracket_{\mathbb{K}}=\left\{\left(\boldsymbol{a}, \mathcal{M}_{e} \boldsymbol{a}\right) \mid \boldsymbol{a} \in \mathbb{K}^{p}\right\}$ ), such that

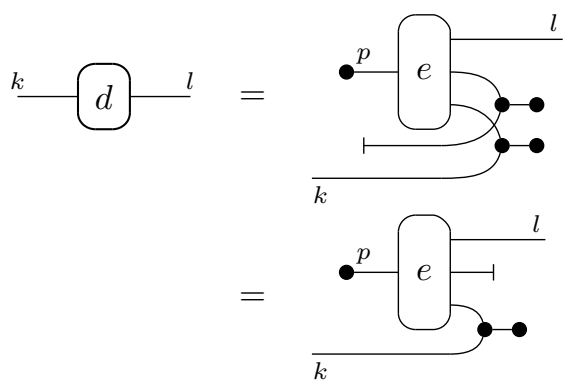

and the columns of $\mathcal{M}_{a}$ generate $\llbracket c^{\prime} \rrbracket_{\mathbb{K}}$ (for the definition of $\longrightarrow$, see (9)). By equation (11) and the isomorphism $\mathrm{IH}_{\mathbb{K}} \cong$ LinRel $_{\mathbb{K}}$, the affine relation $\llbracket d \rrbracket_{\mathbb{K}}$ is uniquely characterised by this decomposition if it is nonempty. If it empty however, it means that $\longrightarrow$ is plugged to a wire of $e$ representing some row of $\mathcal{M}_{e}$ (in the sense of Appendix B) which is uniformly zero. Indeed, if $\left(a_{1} \ldots a_{n}\right)$ is this row, $\llbracket d \rrbracket_{\mathbb{K}}$ is empty precisely when there is no $x_{1}, \ldots, x_{n} \in \mathbb{K}$ such that $a_{1} x_{1}+\cdots+a_{n} x_{n}=$ 1. This happens only when $a_{i}=0$ for all $1 \leq i \leq n$ or, in diagrammatic form, when:

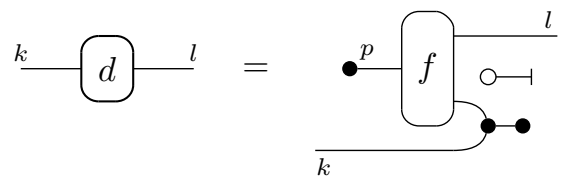

for some diagram $f$ representing a matrix. By Lemma 39 all diagrams of this form are equal, which concludes the proof. 
Corollary 18 (Soundness and Full Completeness). For any two string diagrams $c, d: k \rightarrow l$ in $\mathrm{AlH}_{\mathbb{K}}$,

$$
c=d \text { iff } \llbracket c \rrbracket_{\mathbb{K}}=\llbracket d \rrbracket_{\mathbb{K}} .
$$

and for each $R$ in $\left.A f f R e\right|_{\mathbb{K}}$ there is some e such that $\llbracket e \rrbracket_{\mathbb{K}}=R$.

\section{B. Axiomatising $\mathbb{N}$-affine relations}

The characterisation of $\mathbb{N}$-affine relations requires a bit more work. Analogously to the field case, we take as starting point the equational theory characterising the prop AddRel of (nonaffine) additive relations. This theory is called the algebra of resources (see [13, Fig. 4], reported in Fig. 7, Appendix A). We write RC for the prop obtained by quotienting $\operatorname{Circ}_{\mathbb{N}}$ by its equations.

In order to extend the result to $\mathbb{N}$-affine relations, we need suitable extra equations for $\vdash$. These will include (dup), (del), $(\varnothing)$ from Section IV-A and, because $\mathbb{N}$ misses multiplicative inverses, we require one additional equation:

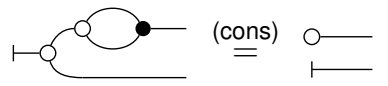

Definition 19. The prop $\mathrm{ARC}$ is the quotient of $\mathrm{ACirc} \mathrm{C}_{\mathbb{N}}$ by the equations of RC (Fig. 7, Appendix A) plus (dup), (del), ( $)$ and (cons).

The new equality (cons) enforces the consistency of systems of non-negative integer equations. In symbolic form it guarantees that, if $2 n+m=1$ then $n=0$ and $m=1$. Note that this is not true when interpreted over a field so that (cons) is not sound for $\left.A f f R e\right|_{\mathbb{K}}$, for any $\mathbb{K}$.

From this simple axiom, we can prove that $p_{1} n_{1}+\cdots+$ $p_{k} n_{k}=1$ has a satisfying assignment if and only if not all the $n_{i}$, for $1 \leq i \leq k$ are strictly greater than 1 . This is a key fact about natural numbers, needed for the characterisation of $\mathbb{N}$ affine relations. The following lemmas express it in graphical form. Their proofs are in Appendix C-A.

Lemma 20. For all $n>1$,

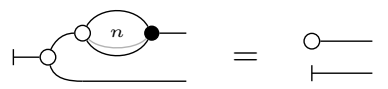

Lemma 21. For $n_{1}, \ldots, n_{k}$ such that $n_{i}>1$ for all $i \leq k$ :

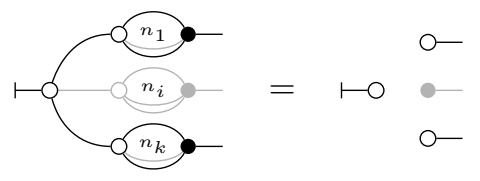

We now have all the ingredients to show that ARC is isomorphic to AffRel $\left.\right|_{\mathbb{N}}$. Because all the equations of AffRel $\left.\right|_{\mathbb{N}}$ are sound for $\mathbb{N}$-affine relations, we can define a prop morphism $\llbracket \cdot \rrbracket_{\mathbb{N}}:$ ARC $\rightarrow$ AffRel $_{\mathbb{N}}$ inductively by the same clauses (Figure 2 ) of $\langle\cdot\rangle_{\mathbb{K}}$.

Theorem 22. $\llbracket \cdot \rrbracket_{\mathbb{N}}:$ ARC $\rightarrow$ AffRel $_{\mathbb{N}}$ is a prop isomorphism.

Proof. The proof of fullness follows the same steps as for Theorem 17, but uses the result, from [13], that the restriction of $\llbracket-\rrbracket_{\mathbb{N}}$ to $R C \rightarrow$ AddRel is an isomorphism (hence, full).
We move on to faithfulness. Again, the first steps of the proof are the same as for Theorem 17: given $d$ in ARC, we can always use the naturality of the symmetry, the (dup) axiom and the normal form for additive relations [13, Section 3.4] to obtain $e: p \rightarrow l+1+k$ representing some matrix $\mathcal{M}_{e}$ (i.e. $\left.\llbracket e \rrbracket_{\mathbb{N}}=\left\{\left(\boldsymbol{a}, \mathcal{M}_{e} \boldsymbol{a}\right) \mid \boldsymbol{a} \in \mathbb{N}^{p}\right\}\right)$ such that

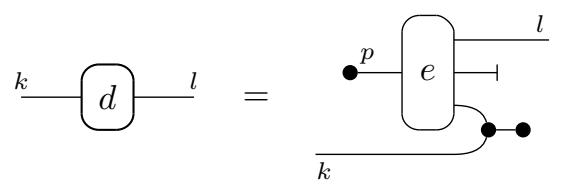

As before, property (11) and the isomorphism of RC and AddRel ( [13]), the $\mathbb{N}$-affine relation $\llbracket d \rrbracket_{\mathbb{N}}$ is uniquely characterised by this decomposition if it is nonempty. The case in which it is empty is slightly more complicated than for $\mathbb{K}$ affine relations. This means that $\longrightarrow$ is plugged to a wire of $e$ representing a row of $\mathcal{M}_{e}$ (in the sense of Appendix B) which sums to a nonnegative integer different from one. Thus it is $\left(\begin{array}{llll}n_{1} & n_{2} & \ldots & n_{k}\end{array}\right)$ with either all $n_{i}>1$ or all zero $\left(n_{i}=0\right.$, for $\left.1 \leq i \leq k\right)$. In the first case, there exists a diagram $f$ representing a matrix, such that

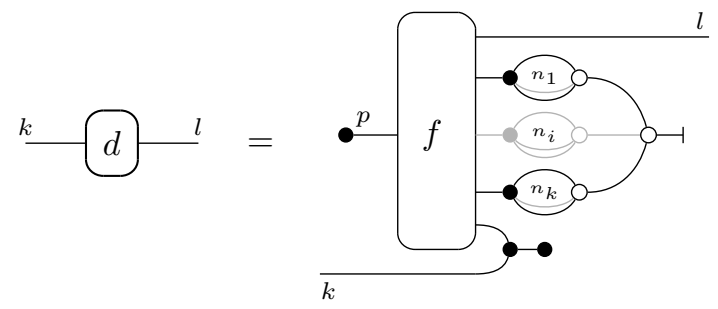

and therefore, by Lemma 41,

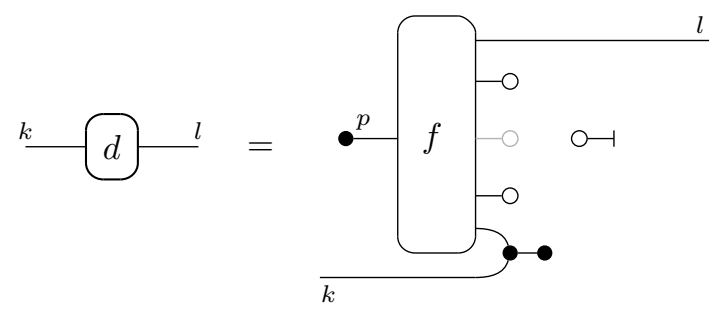

In the second case, $e$ is disconnected from $\longrightarrow$. We can deduce that $d$ is of the following form, for some diagram $f$ representing a matrix:

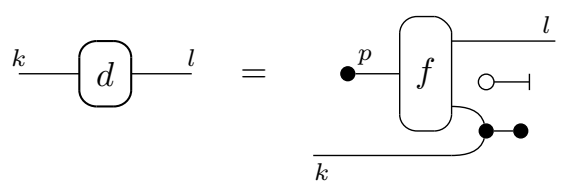

Note that Lemma 39 also holds for ARC (as all the equations required to prove it also hold) and therefore all diagrams of this form are equal, which concludes the proof.

Corollary 23 (Soundness and Full Completeness). For any two string diagrams $c, d: k \rightarrow l$ in ARC,

$$
c=d \text { iff } \llbracket c \rrbracket_{\mathbb{N}}=\llbracket d \rrbracket_{\mathbb{N}}
$$

and for each $A$ in AffRel $_{\mathbb{N}}$ there is some e such that $\llbracket e \rrbracket_{\mathbb{N}}=A$. 


\section{CASe Study I: Stateless Connectors}

While additive relations, via the corresponding diagrammatic theory RC, can express basic forms of synchronisation and parallelism, there are many interesting non-additive phenomena in concurrency that remain out of reach. One notable example is that of mutual exclusion: when two or more processes are prevented from operating or accessing a shared resource at the same time [22]. The key insight of this section is that mutual exclusion is an affine phenomenon. Indeed, as we will see, by moving to ARC we will be able to model not only mutual exclusion, but also the more general inhibitory patterns of synchronisation captured by the calculus of stateless connectors [14]. More specifically, we will show that (i) all behaviour specified by stateless connectors can be expressed, via a compositional semantics-preserving translation, by components from $\mathrm{ACirc}_{2}$ and that (ii) the equational theory of ARC is sound and complete with respect to this translation. Note that, here, $2=\{0,1\}$ denotes the Boolean semiring.

The syntax of stateless connectors is given below, assuming a sorting discipline analogous to that of Section II.

$$
\begin{aligned}
& \left.c, d \quad:=\quad+\bullet\left|+\bullet^{+}\right| \bullet+\mid\right\}+130+\mid \text { o+ }
\end{aligned}
$$

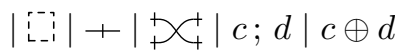

We use crossed wires to indicate that the intuition is different to $\mathrm{ACirc}_{2}$ : the resources passing through the wires are not a natural number, but either 0 or 1 (dubbed, respectively, untick and tick in [14]). Later, we will see that these crossed wires can be understood as diagrams of $\mathrm{ACirc}_{\mathbb{N}}$. A term of (27) specifies a connector that coordinates software components attached to its ports: the signal 1 means that the component is synchronising, 0 that is not. We denote the prop of string diagrams of this syntax by SCCirc.

We focus on the standard denotational meaning [14] of (27) terms via tick tables, which are simply relations $R \subseteq 2^{k} \times 2^{l}$ for $k, l \in \mathbb{N}$. We recall this in Definition 24 below: $\operatorname{Rel}_{2}$ is an instance of Definition 2 and $\bullet$ is the unique element of $2^{0}$.

Definition 24. The prop morphism $\langle\langle-\rangle\rangle: \mathrm{SCCirc} \rightarrow \mathrm{Re}_{2}$ is recursively defined as follows:

$$
\begin{aligned}
& \left\langle\left\langle+\bullet_{+}^{+}\right\rangle\right\rangle=\left\{\left(0,\left(\begin{array}{l}
0 \\
0
\end{array}\right)\right),\left(1,\left(\begin{array}{l}
1 \\
1
\end{array}\right)\right)\right\} \quad\langle\langle-\bullet \bullet\rangle=\{(0, \bullet),(1, \bullet)\} \\
& \left\langle\langle\langle\bullet+\rangle\rangle=\left\{\left(\left(\begin{array}{l}
0 \\
0
\end{array}\right), 0\right),\left(\left(\begin{array}{l}
1 \\
1
\end{array}\right), 1\right)\right\} \quad\langle\langle\bullet-\rangle\rangle=\{(\bullet, 0),(\bullet, 1)\}\right. \\
& \left\langle\left\langle\begin{array}{l}
- \\
+
\end{array}+\right\rangle\right\rangle=\left\{\left(\left(\begin{array}{l}
0 \\
0
\end{array}\right), 0\right),\left(\left(\begin{array}{l}
1 \\
0
\end{array}\right), 1\right),\left(\left(\begin{array}{l}
0 \\
1
\end{array}\right), 1\right)\right\} \\
& \langle\langle o+\rangle\rangle=\{(\bullet, 0)\} \quad\left\langle\left\langle i_{-}^{i}\right\rangle\right\rangle=\{(\bullet, \bullet)\} \\
& \left.\langle\langle+\rangle\rangle=\{(0,0),(1,1)\} \quad\left\langle\langle\rangle_{+}^{+}\right\rangle\right\rangle=\left\{\left(\left(\begin{array}{l}
x \\
y
\end{array}\right),\left(\begin{array}{l}
y \\
x
\end{array}\right)\right) \mid x, y \in 2\right\} \\
& \langle\langle c ; d\rangle\rangle=\langle\langle c\rangle\rangle ;\langle\langle d\rangle\rangle \quad\langle\langle c \oplus d\rangle\rangle=\langle\langle c\rangle\rangle \oplus\langle\langle d\rangle\rangle
\end{aligned}
$$

Similarly to their counterparts in $\operatorname{Circ}_{2},+\bullet_{+}^{+}$and $+\bullet$ (and their mirror images) act as copier and discarder, and o+ as zero. On the other hand, the generator $30+$ implements mutual exclusion: the synchronisation of the right port is only possible with exactly one of the two left ports. This semantic difference is reflected in the equational theory: e.g. (•०-biun) from Figure 7 does not hold.

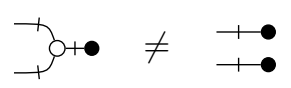

Nevertheless, the semantics of $\mathrm{O}+$ is a $\mathbb{N}$-affine relation, corresponding to the interpretation of the diagram below, already encountered in Section II-D (c).

$$
\begin{gathered}
\langle\bigcirc-\rangle_{\mathbb{N}}=\left\{\left(\left(\left(\begin{array}{c}
1 \\
0
\end{array}\right), 1\right),\left(\left(\begin{array}{l}
0 \\
1
\end{array}\right), 1\right),\left(\left(\begin{array}{l}
0 \\
0
\end{array}\right), 0\right)\right\}\right.
\end{gathered}
$$

The subterm - , which also featured in Section II-D and for which we will use the shorthand $-\_-$, is essential to our translation of SCCirc to ARC. Notice that the ARC diagram denoting mutual exclusion is the composition of 3 with $\longrightarrow$, defined as

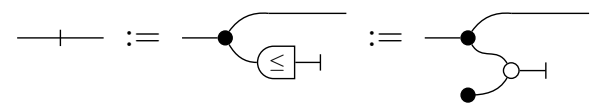

The intuition is that — 1 restricts the bandwidth of the wire, so that it can carry at most one unit of resource. Each generator of SCCirc can now be encoded as the corresponding one of ARC composed with 1 . A key property of -1 is its idempotency.

Proposition 25. $-1,=-$ in ARC.

Proof. Appendix C-B.

Definition 26. The translation $\mathcal{E}(-)$ : SCCirc $\rightarrow$ ARC is defined recursively as follows.

$$
\begin{aligned}
& \mathcal{E}(+\bullet)=+\bullet \\
& \mathcal{E}(+\infty)= \\
& \mathcal{E}\left({ }_{+}^{+}++\right)=\mathrm{O}+ \\
& \mathcal{E}(\bullet+)=\bullet-+ \\
& \mathcal{E}\left(+\boldsymbol{\sigma}_{+}^{+}\right)=+\boldsymbol{c} \\
& \mathcal{E}(\mathrm{o}+)=\mathrm{o}+
\end{aligned}
$$

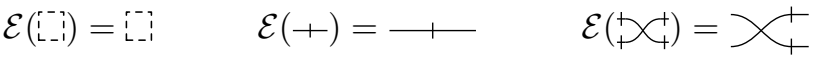

$$
\begin{aligned}
& \mathcal{E}(c ; d)=\mathcal{E}(c) ; \mathcal{E}(d) \quad \mathcal{E}(c \oplus d)=\mathcal{E}(c) \oplus \mathcal{E}(d)
\end{aligned}
$$

Because the identity of SCCirc is mapped to -1 and not to the identity in ARC, we do not have a prop morphism. In fact, for this reason, this encoding is not a functor. One can think of it as a functor up-to an idempotent ${ }^{1}$, representing the inclusion of the subset $\{0,1\} \subseteq \mathbb{N}$.

Theorem 27. Let $\iota_{1}:$ AffRel $_{\mathbb{N}} \rightarrow \operatorname{Re}_{\mathbb{N}}$ be the obvious prop morphism embedding $\mathbb{N}$-affine relations into $\operatorname{Re}_{\mathbb{N}}$ and $\iota_{2}: \operatorname{Re}_{2} \rightarrow \operatorname{Re}_{\mathbb{N}}$ be the mapping arising from the inclusion

\footnotetext{
${ }^{1}$ It is possible to make this notion precise using the idempotent completion (or Karoubi envelope) of ARC. For details, see [23]
} 
$2 \subseteq \mathbb{N}$, interpreting a relation over 2 as a relation over $\mathbb{N} .{ }^{2}$ For all $c$ in SCCirc, the diagram below commutes.

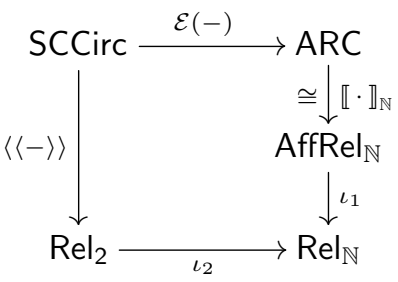

Proof. By induction on SCCirc.

As a consequence of Theorem 27 we obtain a sound and complete axiomatisation for equivalence of stateless connectors by means of the axioms of ARC, in Figure 7.

Corollary 28. For any two stateless connectors $c$ and $d$ in SCCirc,

$$
\langle\langle c\rangle\rangle=\langle\langle d\rangle\rangle \quad \text { iff } \quad \mathcal{E}(c)=\mathcal{E}(d)
$$

Remark 29. Theorem 26 in [14] states that the connectors in SCCirc can denote exactly those relations in $\mathrm{Re}_{2}$ that contain the vector 0 . ACirc 2 can express more relations of $\mathrm{Re}_{2}$, for instance the not relation denoted by the following diagram:

$$
\left.\langle\cdot\rangle_{0}\right\rangle_{\mathbb{N}}=\{(0,1),(1,0)\}
$$

In fact, all relations in $\mathrm{Rel}_{2}$ can be expressed by $\mathrm{ACirc}_{2}$ since every finite subset of $\mathbb{N}^{k} \times \mathbb{N}^{l}$ is an $\mathbb{N}$-affine relation $k \rightarrow l$, so in particular every subset containing only $0 s$ and $1 s$ is in AffRel $_{\mathbb{N}}$.

\section{Case Study II: Electric Circuits}

Elementary electrical engineering focusses on open linear circuit analysis. An example is illustrated below.

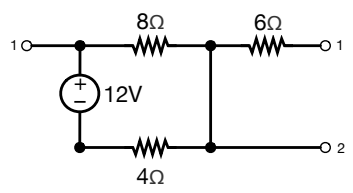

Such circuits may include voltage $(-\odot)$ and current sources

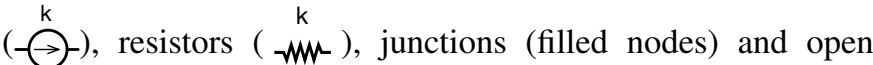
terminals (unfilled nodes).

The section is structured as follows. We begin by making these open circuits formal as combinatorial structures. We then present open circuits as algebraic structures, and give a compositional semantics in terms of $\mathbb{K}$-affine relations. In Subsection VI-A, we use the axiomatisation of Section IV to give a sound and complete calculus for the analysis of open linear circuits. We end in Subsection VI-B by showing how to handle circuits with time-dependent currents and voltages, which also feature inductors $(\stackrel{\mathrm{k}}{\mathrm{m}})$ and capacitors $(\stackrel{\mathrm{k}}{-1})$.

\footnotetext{
${ }^{2}$ Note that $\iota_{2}$ is not a functor since it does not preserve identities.
}

We can make closed (i.e. those without open terminals) circuits precise as combinatorial structures by considering them as multigraphs with a mixture of directed and undirected edges. Directed edges are either voltage and current sources, while undirected edges are resistors. Finally, every edge is labelled by a non-negative real, denoting either voltage (in volts), current (in amperes) or resistance (in ohms). Formally, then, a closed circuit is

$$
\begin{array}{r}
\{X, V, C, R, v s, v t: V \rightarrow X, c s, c t: C \rightarrow X \\
\left.r c: R \rightarrow \mathcal{P}_{2}(X), q: V+C+R \rightarrow \mathbb{R}_{+}\right\}
\end{array}
$$

where $X, V, C, R$ are, correspondingly, finite sets of nodes, voltage sources, current sources and resistors, $v s, v t, c s, c t, r c$ give the connectivity of the edges, and $q$ the labels.

To consider open circuits, we consider a certain category of cospans. First, the category CCirc of closed circuits and their obvious choice of morphism has pushouts. Next, any finite ordinal can be considered as a discrete closed circuit, with the ordinal serving as its set of nodes. We therefore consider the full subcategory OCirc of the category of cospans Cospan (CCirc) with objects finite ordinals. Having ordinals as objects reflects the numbering the left and right open terminals, as we have done in the example diagram above. It is straightforward to verify that OCirc is a prop.

We now give a straightforward algebraic characterisation of OCirc. The prop ECirc is has signature

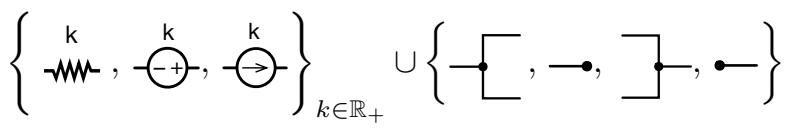

where the parameter $k$ ranges over the non-negative reals. Arrows $m \rightarrow n$ of ECirc represent open linear electrical circuits with $m$ open terminals on the left and $n$ open terminals on the right. The following are the equations:

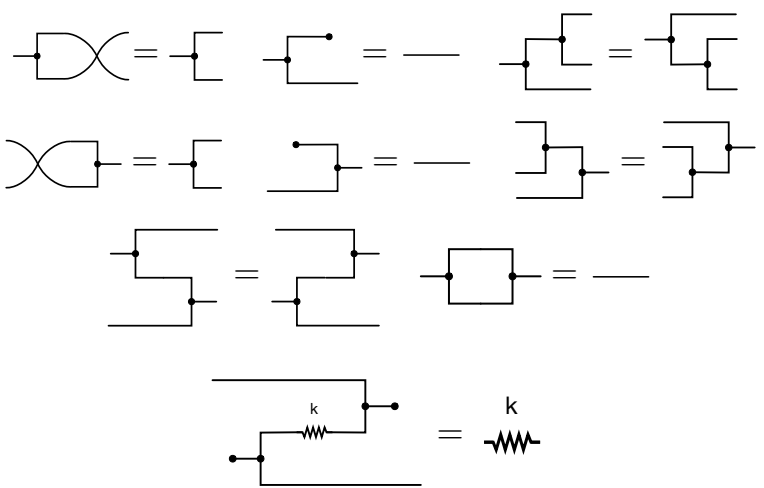

The equations, apart from the last, are those of special Frobenius monoids [24]. The final equation reflects the fact that resistors are bidirectional. We state the following without proof, which is similar to [25, Proposition 3.2] and [26, Theorem 3.3].

Proposition 30. As props, OCirc $\cong$ ECirc.

Having established open circuits as both combinatorial (OCirc) and algebraic (ECirc) structures, we can now give 


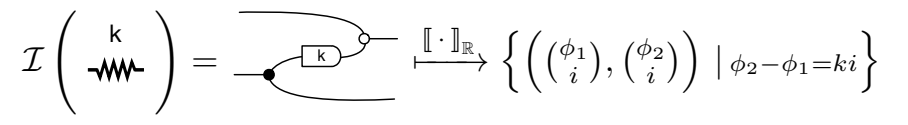

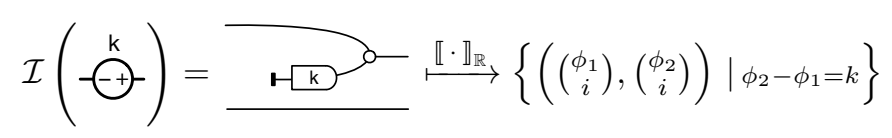

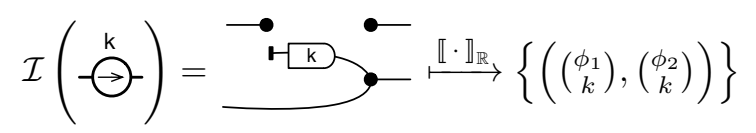

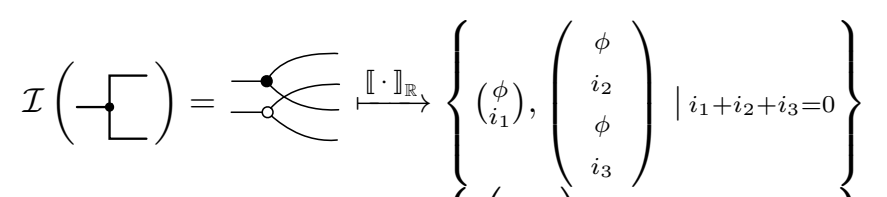

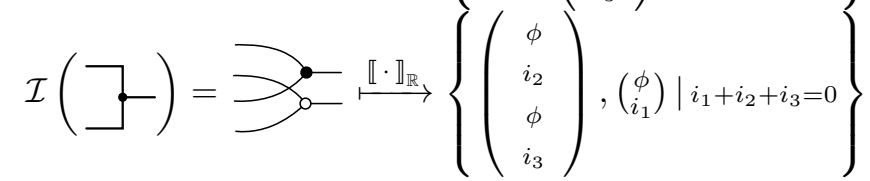

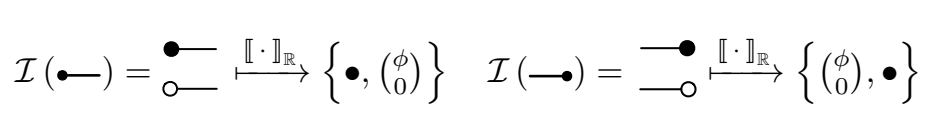

Fig. 5. Compositional semantics of electrical circuits.

a compositional semantics in terms of affine relations in Fig. VI. For each generator we give its translation as $\mathrm{AlH}_{\mathbb{R}}$-diagram and the associated $\mathbb{R}$-affine relation in symbolic notation. A similar semantics was given by Baez and Coya [15], [27], building on the work of Baez, Erbele and Fong [7], [28], and Rosebrugh, Sabadini and Walters [25]. Components of an electrical circuit denote a relationship between current and voltage, traditionally modelled as real values. The semantics of an open circuit of type $m \rightarrow n$ is thus a relation from $\mathbb{R}^{2 m}$ to $\mathbb{R}^{2 n}$, with the behaviour of the individual elements given by Kirchoff's laws. We use $\phi \in \mathbb{R}$ to range over voltages and $i \in \mathbb{R}$ to range over currents.

Proposition 31. $\mathcal{I}(-):$ ECirc $\rightarrow$ AffRel $_{\mathbb{R}}$ is a symmetric strict monoidal functor.

Proof. See Appendix C-B.

Note that $\mathcal{I}(-)$ fails to be a morphism of props for the simple reason that it is not identity on objects: a single wire of an electrical circuit maps to two wires of $\mathrm{AlH}_{\mathbb{R}}$, with these used to keep track of the voltage and the current. Indeed, on objects $\mathcal{I}(1)=2$.

\section{A. $\mathrm{AlH}_{\mathbb{R}}$ as a Calculus of Electrical Circuits}

Below we give a few examples of how $\mathrm{AlH}_{\mathbb{R}}$ can be used to derive well-known properties of circuits.

Lemma 32 (Properties of resistors).

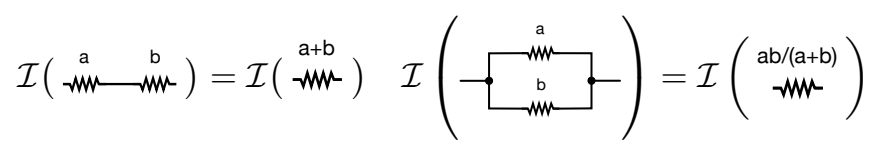

Proof. See Appendix C-B.
Lemma 33 (Properties of voltage sources).

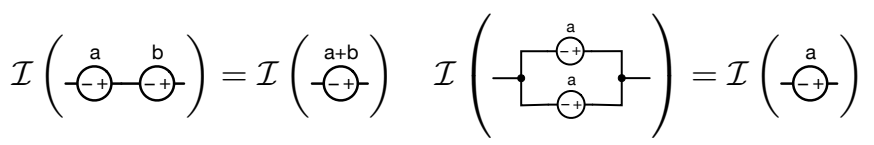

Proof. We only prove the first equality.

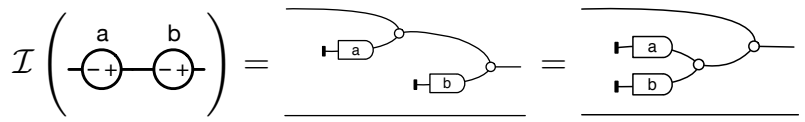

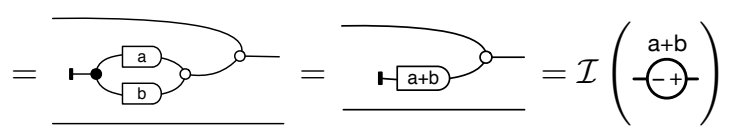

Remark 34. In engineering literature, parallel voltage sources of different voltages are disallowed. It is nonetheless interesting to see what happens in the semantics.

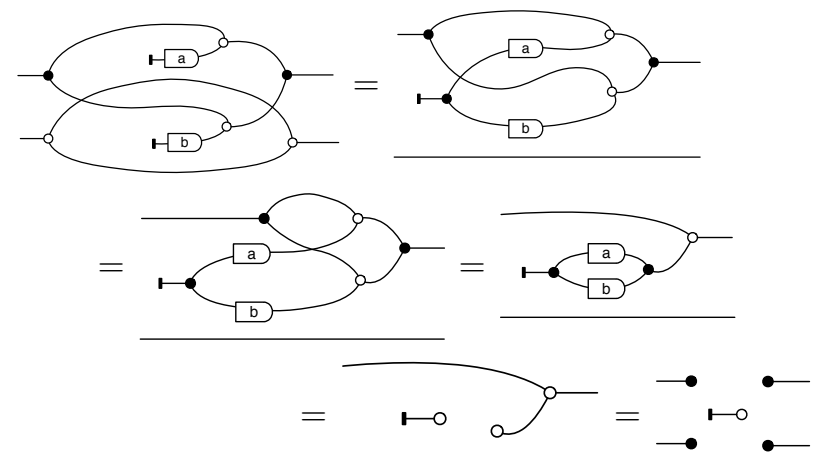

This, as we have seen, is the way of expressing the empty relation in graphical affine algebra.

Lemma 35 (Properties of current sources).

$$
\mathcal{I}\left(-\bigodot^{\mathrm{a}}-\stackrel{\mathrm{a}}{\ominus}\right)=\mathcal{I}\left(-^{\mathrm{a}}-\right) \quad \mathcal{I}\left(-\stackrel{\Theta^{\mathrm{b}}}{\Theta}-\leftarrow\right)=\mathcal{I}\left(--^{\mathrm{a}+\mathrm{b}}-\right)
$$

Proof. We prove only the second equality.

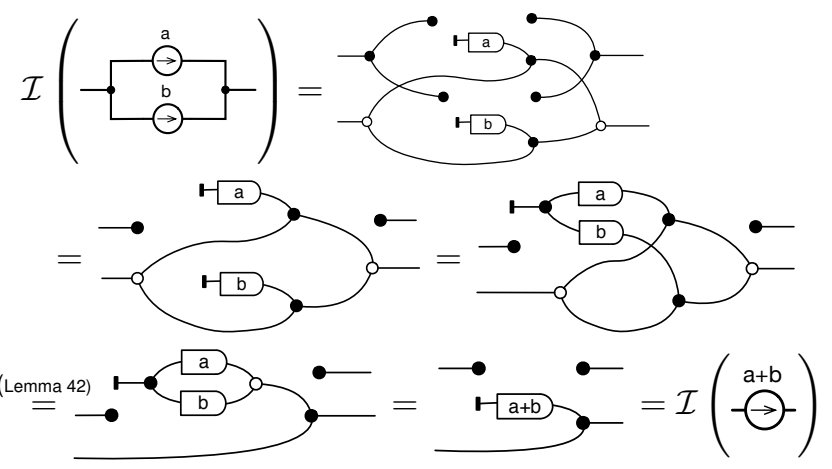

Remark 36. Just as different voltage sources cannot be put in parallel (Remark 34), different current sources cannot be put in series: a similar graphical calculation as in Remark 34 yields the empty relation. 


\section{B. From $\mathbb{R}$ to $\mathbb{R}(x)$ : Inductors and Capacitors}

To capture time-dependent currents and voltages, we extend circuits with two additional kinds of undirected edges, induc-

tors $\stackrel{k}{m}$ and capacitors $-1-$, each with labels from $\mathbb{R}_{+}$, signifying inductance and capacitance. Omitting the details of the straightforward formalisation, we obtain $\mathbf{C C i r c}_{s}$ by considering the category of such extended circuits and, via cospans, the corresponding category of open circuits $\mathbf{O C i r c}_{s}$.

To obtain ECirc $s$ we extend the signature (28) with inductors and capacitors $\left\{\begin{array}{cc}k & k \\ m & -1-\end{array}\right\}_{k \in \mathbb{R}_{+}}$and extend the set equations of ECirc with those that indicate that these additional elements are undirected
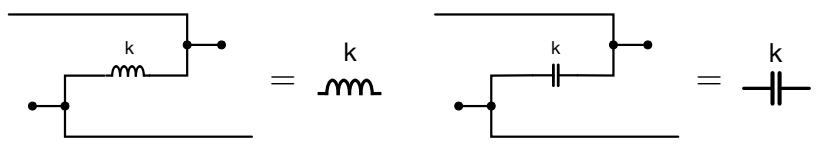

The following is a simple extension of the correspondence shown in Proposition 30.

Proposition 37. As props, $\mathrm{OCirc}_{s} \cong \mathrm{ECirc}_{s}$.

By moving from the reals $\mathbb{R}$ to the field of polynomial fractions $\mathbb{R}(x)$, or equivalently, rational functions in one variable, we can give a compositional semantics of circuits with time-dependent currents and voltages. The idea is to let multiplication by $x$ express differentiation by the time variable, as usually done in engineering via Laplace transforms. We extend the mapping of Figure 5 as follows:

$$
\left.\begin{array}{l}
\mathcal{I}(\mathrm{m} \\
\mathrm{m}
\end{array}\right)=\longrightarrow
$$

We can then show that also the extended semantics is functorial.

Proposition 38. $\mathcal{I}(-): \mathrm{ECirc}_{s} \rightarrow \operatorname{AffRel}_{\mathbb{R}(x)}$ is a symmetric strict monoidal functor.

Proof. Follows from the proof of Proposition 44. It suffices to check that the undirectedness of $\mathrm{m}$ and $\stackrel{k}{-1}$ is respected, but the derivation given in the proof of Proposition 44 can easily be adapted in each case.

Exploiting the isomorphism $\mathrm{AlH}_{\mathbb{R}(x)} \cong \operatorname{AffRel}_{\mathbb{R}(x)}$, we can reason equationally also on this extended class of circuits. For instance, one can show that inductors behave analogously to resistors when put in series and in parallel- $c f$. Lemma 32 and Appendix C-B, Lemma 46. This is mostly evident when observing the structural similarity of their $\mathrm{AlH}_{\mathbb{R}(x)}$-interpretation:

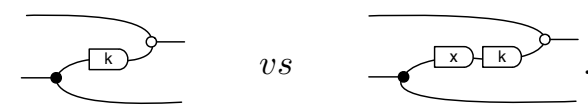

\section{REFERENCES}

[1] E. W. Dijkstra, "Cooperating sequential processes," in The origin of concurrent programming. Springer, 1968, pp. 65-138.

[2] S. Abramsky, "What are the fundamental structures of concurrency? we still don't know!" arXiv:1401.4973, 2014.

[3] F. Bonchi, P. Sobociński, and F. Zanasi, "Interacting Hopf algebras," $J$ Pure Appl Alg, vol. 221, no. 1, pp. 144-184, 2017.

[4] F. Zanasi, "Interacting hopf algebras: the theory of linear systems," Ph.D. dissertation, Ecole Normale Supérieure de Lyon, 2015.

[5] F. Bonchi, P. Sobociński, and F. Zanasi, "Interacting bialgebras are Frobenius," in FOSSACS '14, pp. 351-365.

[6] B. Coecke and R. Duncan, "Interacting quantum observables," in ICALP '08, pp. 298-310.

[7] J. Baez and J. Erbele, "Categories in control," TAC 30:836-881, 2015.

[8] F. Bonchi, P. Sobocinski, and F. Zanasi, "Full abstraction for signal flow graphs," in POPL '15, pp. 515-526.

[9] B. Fong, P. Rapisarda, and P. Sobociński, "A categorical approach to open and interconnected dynamical systems," in LICS '16.

[10] C. E. Shannon, "The theory and design of linear differential equation machines," National Defence Research Council, Tech. Rep., 1942.

[11] S. J. Mason, Feedback Theory: I. Some Properties of Signal Flow Graphs. MIT Research Laboratory of Electronics, 1953.

[12] J. C. Willems, "The behavioural approach to open and interconnected systems," IEEE Contr Syst Mag, vol. 27, pp. 46-99, 2007.

[13] F. Bonchi, J. Holland, R. Piedeleu, P. Sobocinski, and F. Zanasi, "Diagrammatic algebra: from linear to concurrent systems," in POPL ' 19.

[14] R. Bruni, I. Lanese, and U. Montanari, "A basic algebra of stateless connectors," Theor Comput Sci, vol. 366, no. 1-2, pp. 98-120, 2006.

[15] J. C. Baez, B. Coya, and F. Rebro, "Props in network theory," arXiv:1707.08321, 2017.

[16] F. Arbab, R. Bruni, D. Clarke, I. Lanese, and U. Montanari, "Tiles for reo," in WADT '08, pp. 37-55.

[17] R. Bruni, H. Melgratti, and U. Montanari, "Connector algebras, Petri nets, and BIP," in Andrei Ershov Memorial Conf, 2011, pp. 19-38.

[18] F. Bonchi, P. Sobocinski, and F. Zanasi, "A categorical semantics of signal flow graphs," in CONCUR '14, pp. 435-450.

[19] — "The calculus of signal flow diagrams I: linear relations on streams," Inf Comput 252:2-29, 2017.

[20] P. Selinger, "A survey of graphical languages for monoidal categories," Springer Lecture Notes in Physics, vol. 13, no. 813, pp. 289-355, 2011.

[21] G. M. Kelly and M. L. Laplaza, "Coherence for compact closed categories," J Pure Appl Alg, vol. 19, pp. 193-213, 1980.

[22] E. Dijkstra, "Solution of a problem in concurrent programming control," Communications of the ACM, vol. 8, no. 9, p. 569, 1965.

[23] R. Piedeleu, "Picturing resources in concurrency," Ph.D. dissertation, University of Oxford, 2018.

[24] A. Carboni and R. F. C. Walters, "Cartesian bicategories I," J Pure Appl Alg 49:11-32, 1987.

[25] R. Rosebrugh, N. Sabadini, and R. F. C. Walters, "Generic commutative separable algebras and cospans of graphs," TAC 17(6):164-177, 2005.

[26] F. Bonchi, F. Gadducci, A. Kissinger, P. Sobocinski, and F. Zanasi, "Rewriting modulo symmetric monoidal structure," in LICS '16.

[27] B. Coya, "Circuits, bond graphs, and signal-flow diagrams: A categorical perspective," Ph.D. dissertation, U California Riverside, 2018.

[28] J. C. Baez and B. Fong, "A compositional framework for passive linear networks," arXiv:1504.05625, 2015.

[29] S. Lack, "Composing PROPs," TAC 13(9):147-163, 2004.

[30] J. Kock, Frobenius algebras and $2 D$ topological quantum field theories. CUP, 2003.

[31] B. Coecke and A. Kissinger, Picturing Quantum Processes - A first course in Quantum Theory and Diagrammatic Reasoning. CUP, 2017.

[32] R. Bruni and F. Gadducci, "Some algebraic laws for spans (and their connections with multi-relations)," in RelMiS 2001. Elsevier, 2001.

[33] F. Zanasi, "The algebra of partial equivalence relations," in MFPS '16, pp. 313-333.

[34] B. Coya and B. Fong, "Corelations are the prop for extraspecial commutative Frobenius monoids," TAC 32(11):280-395, 2017.

[35] B. Coecke, D. Pavlovic, and J. Vicary, "A new description of orthogonal bases," Math Struct Comp Sci, vol. 23, no. 3, pp. 557-567, 2012.

[36] F. Bonchi, D. Pavlovic, and P. Sobocinski, "Functorial semantics for relational theories," arXiv:1711.08699, 2017.

[37] B. Coecke and R. Duncan, "Interacting quantum observables: categorical algebra and diagrammatics," New J Physics 13(4):043016, 2011. 


\section{APPENDIX A}

AXIOMATISATIONS FOR NON-AFFINE CIRCUIT DIAGRAMS

In this appendix we report from [3] and [13] the equational theories axiomatising (non-affine) linear relations and additive relations, called the theory of interacting Hopf algebras $\left(\mathrm{IH}_{\mathbb{K}}\right)$ and the algebra of resources (RC) respectively. For each theory, we provide a brief description of its equations.

\section{A. Interacting Hopf algebras}

The following description refers to Figure 6.

- In the first block, both the black and white structures are commutative monoids and comonoids, expressing fundamental properties of addition and copying.

- In the second block, the white monoid and black comonoid interact as a bimonoid. Bimonoids are one of two canonical ways that monoids and comonoids interact, as shown in [29].

- In the third and fourth block, both the black and the white monoid/comonoid pair form an extraspecial Frobenius monoid. The Frobenius equations ( $f r 1$ ) and ( $f r 2$ ) are a famous algebraic pattern which establishes a bridge between algebraic and topological phenomena, see [24], [30], [31]. The "extraspecial" refers to the two additional equations, the special equation $(\bullet-\mathrm{sp})$ and the bone equation $(\bullet-b o)$. The Frobenius equations, together with the special equation, are the another canonical pattern of interaction between monoids and comonoids identified in [29]. Together with the bone equation, the set of four equations characterises corelations, see [32]-[34].

- The equations in the fifth block are parametrised over $r \in \mathbb{K}$ and describe commutativity of $-k$ - with respect to the other operations, as well as multiplication and addition of scalars.

- Finally, the last block describes the interpretation of -k- as division by $k$.

\section{B. The algebra of resources}

The following description refers to Figure 7.

- The first three blocks are the same as in $\mathrm{IH}_{\mathbb{K}}, c f$. Figure 6.

- In the fourth block, deviating from the equational theory of $\mathrm{IH}_{\mathbb{K}}$, the white monoid-comonoid pair forms a special bimonoid, not a Frobenius monoid. In fact, the Frobenius structure is equivalent to the presence of additive inverses [35], [36] and it is incompatible with the nonnegativity of the natural numbers: $a+b=0 \Longrightarrow a=$ $b=0$, reflected by (o-bi(co)un).

- In the fifth block, two more equations capture key properties of addition in $\mathbb{N}$. Note that the second can be seen as a unary version of the first. In particular, (can) is one of the two equations that axiomatises the notion of complementary observables in categorical approaches to quantum mechanics [37].

- Finally, the last equation is an axiom scheme, parametrised over $n \in \mathbb{N}$. It uses the following syntactic sugar, along with the obvious mirror image versions, defined recursively:

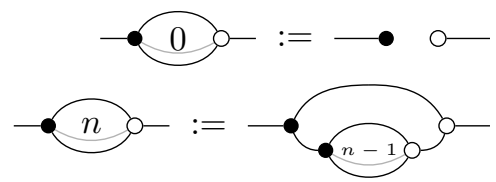

to represent the additive relations of the form $\langle(1, n)\rangle$. Equations ( $n$-inv) are one half of the equations that concerns such sugars in $\mathbb{I H}_{\mathbb{K}}$. Their symmetric variant is not present since they are not sound for AddRel and rely on the presence of multiplicative inverses, which $\mathbb{N}$ does not have.

\section{APPENDIX B \\ PICTURING MATRICES}

The proofs of completeness of $\mathrm{AlH}_{\mathbb{K}}$ (Theorem 17) and ARC (Theorem 22) exploit the ability to represent matrices in the graphical syntax. Details can be found in $[4, \mathrm{Sec}$. 3.2] but we recall the basics below.

Roughly speaking, the theory of matrices lives inside both $\mathrm{AlH}_{\mathbb{K}}$ and ARC as the theory of the bimonoid $-\prec,-, \quad-, \circ-$ along with that of the scalars $-k-$. It means that, using only these we can represent any matrix with coefficients in $\mathbb{K}$ or $\mathbb{N}$. And, moreover, reasoning about them can be done entirely graphically, as the corresponding equational theories are complete.

To develop some intuition for this correspondence, let us demonstrate how matrices are represented diagrammatically. An $l \times k$ matrix $\mathcal{M}_{d}$ corresponds to a diagram $d$ with $k$ wires on the left and $l$ wires on the right - the left ports can be interpreted as the columns and the right ports as the rows of $\mathcal{M}_{d}$. The left $j$ th port is connected to the $i$ th port on the right through an $r$-weighted wire whenever coefficient $\left(\mathcal{M}_{d}\right)_{i j}$ is a nonzero scalar $r \in \mathrm{R}$. When the $\left(\mathcal{M}_{d}\right)_{i j}$ entry is 0 , they are disconnected. Since composition along a wire carries the multiplicative structure of R, we can simply draw the connection as a plain wire if $\left(\mathcal{M}_{d}\right)_{i j}=1$. For example,

$$
\text { The matrix } \mathcal{M}_{d}=\left(\begin{array}{lll}
a & 0 & 0 \\
b & 0 & 1 \\
1 & 0 & 0 \\
0 & 0 & 0
\end{array}\right)
$$

is represented by the following diagram:

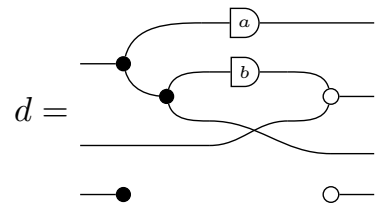

Conversely, given a diagram, we recover the matrix by counting weighted paths from left to right ports. In terms of the isomorphisms $\llbracket \cdot \rrbracket_{R}$ of Section IV, we have that $\llbracket d \rrbracket_{R}=$ $\left\{\left(\boldsymbol{a}, \mathcal{M}_{d} \boldsymbol{a} \mid \boldsymbol{a} \in \mathrm{R}^{k}\right\}\right.$. 


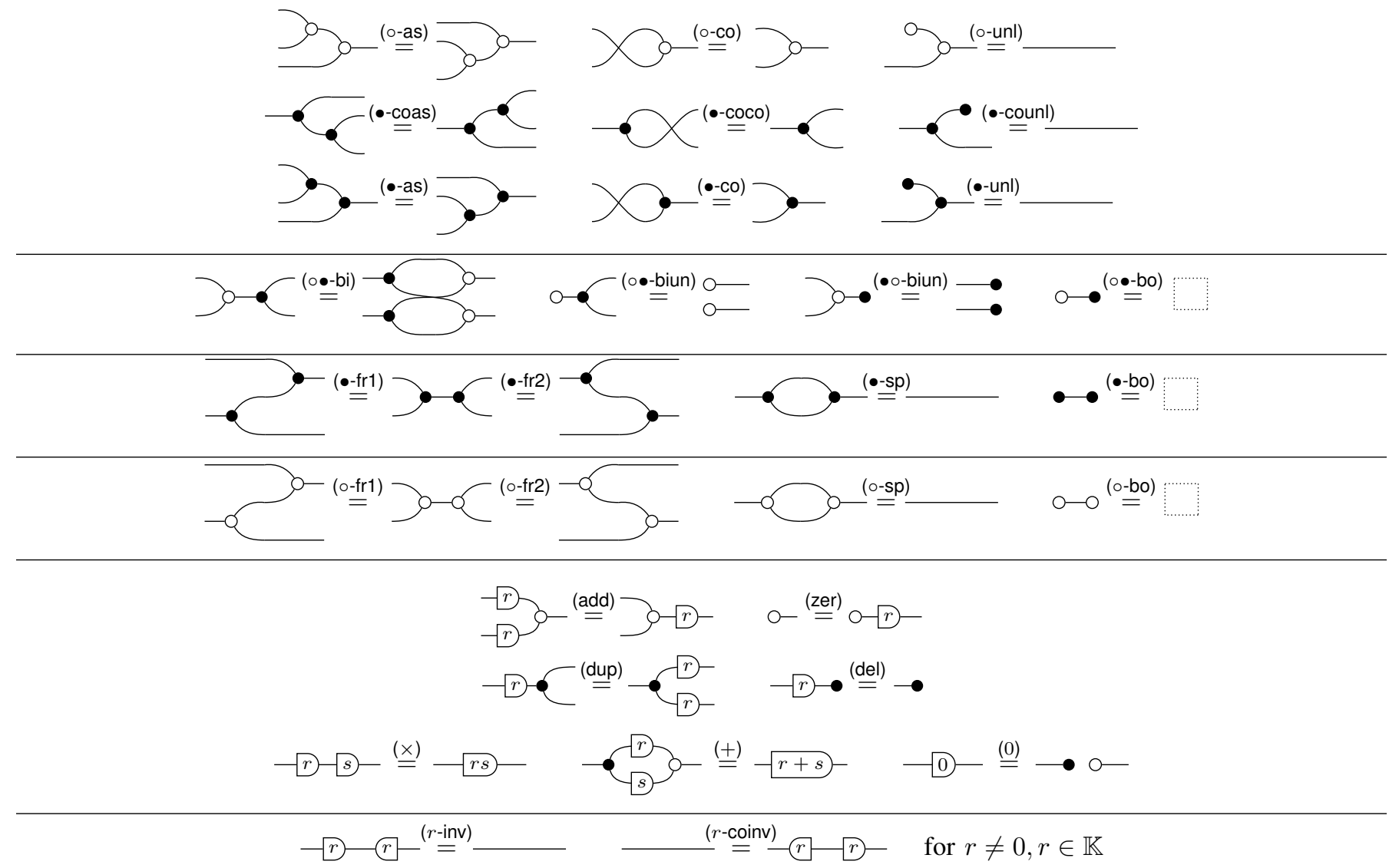

Fig. 6. Axioms of Interacting Hopf Algebras $\left(\mathrm{IH}_{\mathbb{K}}\right)$.

APPENDIX C

MisCELLANEOUS PROOFS

\section{A. Axiomatising Affine Relations}

Lemma 39. For any two arrows $c, d: k \rightarrow l$ of $\mathrm{AlH}_{\mathbb{K}}$,

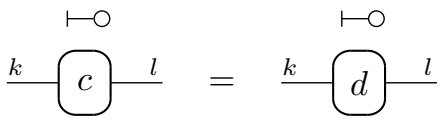

Proof. Because they represent the empty relation, all diagrams of this form should be equal in $\mathrm{AlH}_{\mathbb{K}}$. The proof relies on the ability to completely disconnect all diagrams using the $(\varnothing)$ axiom. To verify this, we can reason by structural induction. For the base cases, we check that all generators of the same type, tensored with $\vdash$, are equal.

- For the counits, we have

$$
\stackrel{\mapsto \circ}{\longrightarrow} \stackrel{(\varnothing)}{=} \stackrel{\bullet}{\bullet} \stackrel{(\text { bo) }}{=} \stackrel{\mapsto \circ}{\longrightarrow}
$$

- For the monoids, we have

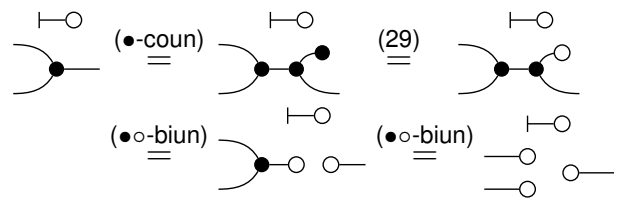

and, furthermore

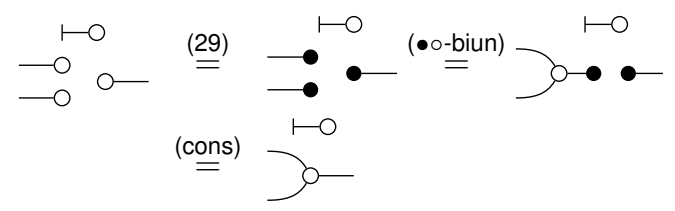

- The reasoning for the dual generators is the same, flipped horizontally.

For the inductive case, notice that

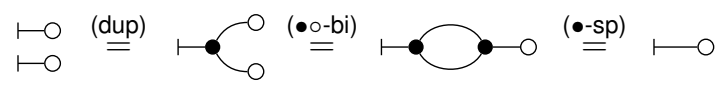




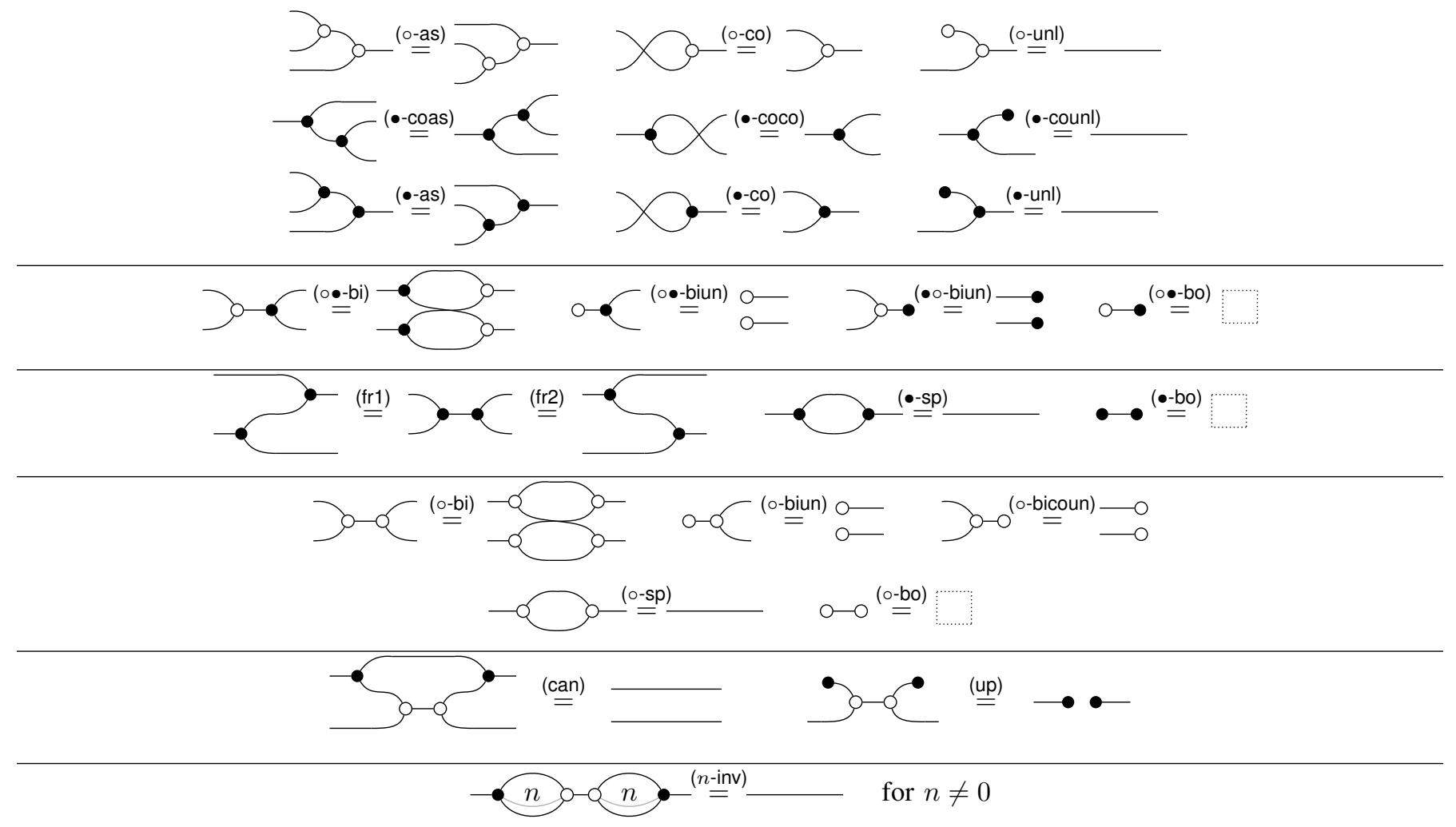

Fig. 7. Axioms of the algebra of resources (RC).

so that their composition satisfies the expected property:

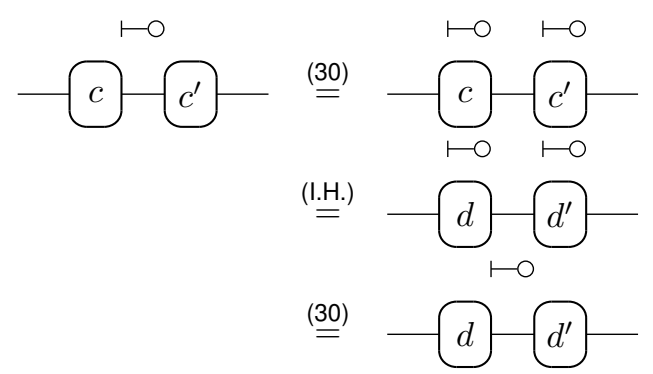

The case of the monoidal product is entirely analogous.

Lemma 40. For all $n>1$,

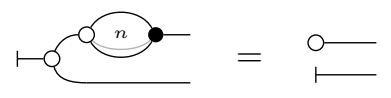

Proof. By induction on $n$. The base case is the axiom (cons). Assume that the statement of the lemma is true for some integer $n>1$. Then,

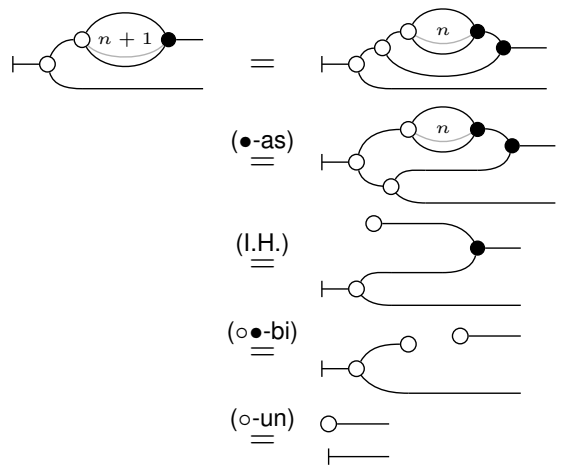

Lemma 41. For $n_{1}, \ldots, n_{k}$ such that $n_{i}>1$ for all $i \leq k$ :

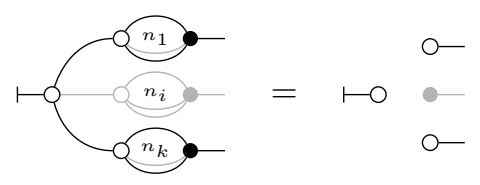

Proof. By induction on $k$. For $k=1$ it is a direct consequence of Lemma 40:

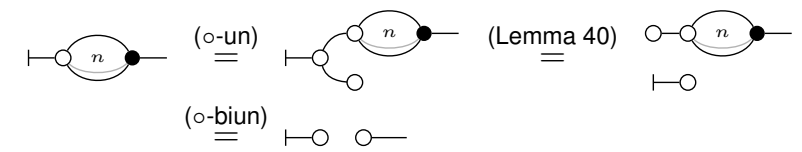


Assume that the statement of the lemma holds for some $k$. Proof.

Then,
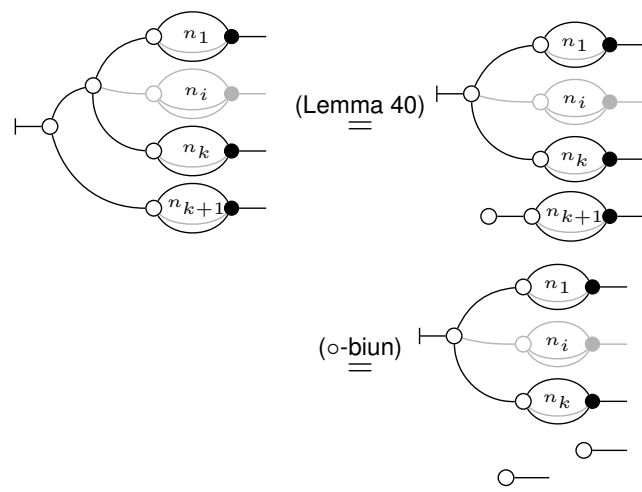

$$
\stackrel{\text { (I.H.) }}{=} \mapsto \mathrm{O}
$$$$
\text { O- }
$$

\section{B. Missing Proofs for the Case Studies}

The following is a useful graphical result.

Lemma 42. In $\mathrm{IH}_{\mathbb{K}}$ and $\mathrm{ARC}$, we have
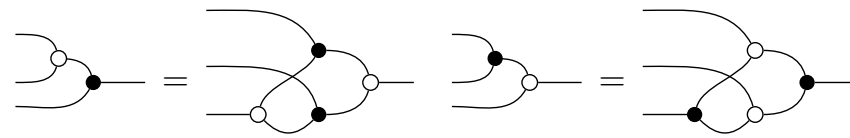

Proof. We prove only one of the equalities.

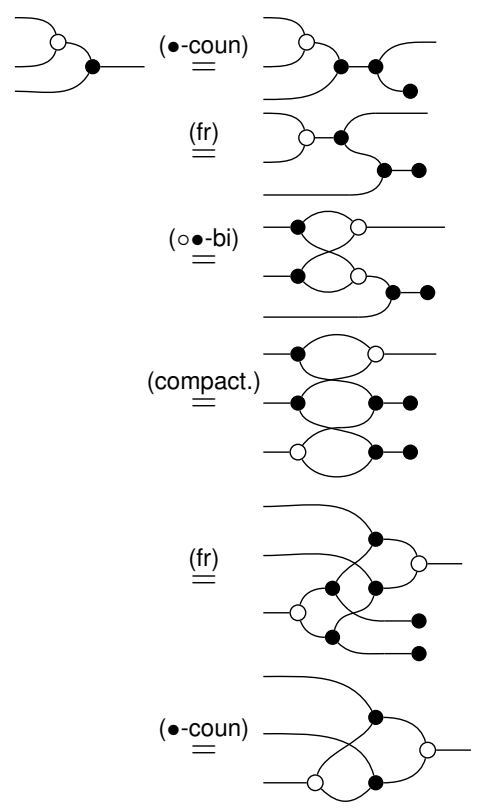

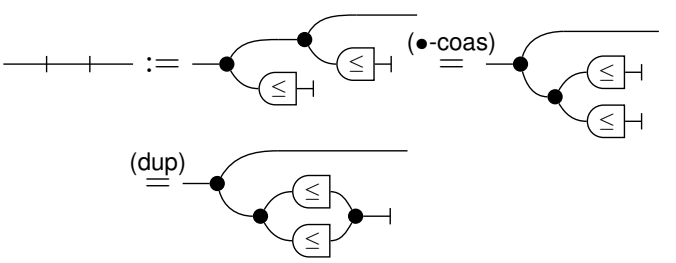

It is now enough to show that

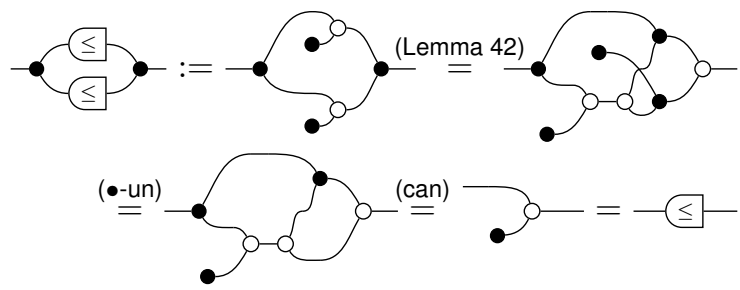

Proposition 44. $\mathcal{I}(-):$ ECirc $\rightarrow$ AffRel $_{\mathbb{R}}$ is a symmetric strict monoidal functor.

Proof. It suffices to show that the mapping is well-defined, i.e. that the equations of ECirc are respected by the semantics: whenever $t=u$ in ECirc then $\mathcal{I}(t)=\mathcal{I}(u)$. The equations of special Frobenius algebras obviously hold, since we are working over $\mathrm{AlH}_{\mathbb{R}}$. The final equation, expressing the bidirectionality of resistors,

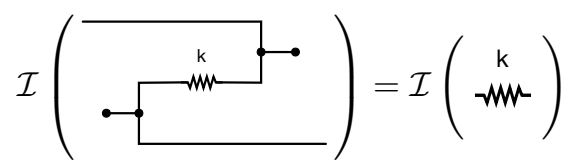

can be checked as follows. First, it is an easy exercise of graphical linear algebra to show that

Proposition 43. in $\mathrm{ARC}$.

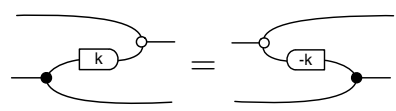


Then, we have

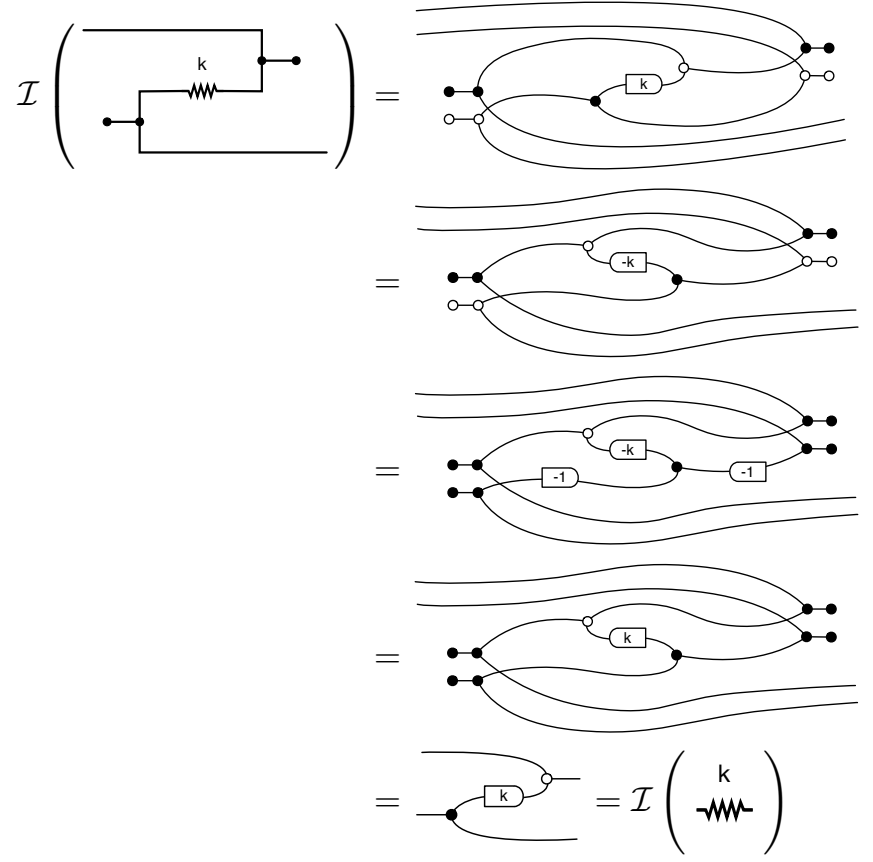

where the penultimate step follows from compactness (see Section II-C).

Lemma 45 (Properties of resistors).

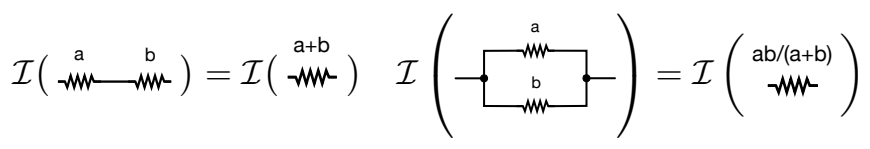

Proof.

$$
\begin{aligned}
& \mathcal{I}\left(\begin{array}{cc}
a & b \\
-m & w
\end{array}\right)= \\
& = \\
& =a_{b} \\
& =\overbrace{}^{-2}=\mathcal{I}\left(\begin{array}{c}
a+b \\
-w h
\end{array}\right)
\end{aligned}
$$
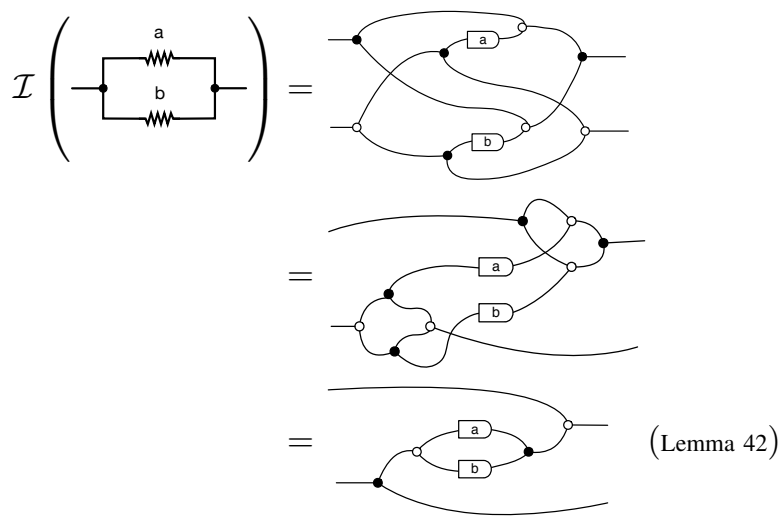

$=\mathcal{I}\left(\begin{array}{c}a b /(a+b) \\ -w\end{array}\right)$

Lemma 46. [Properties of inductors]

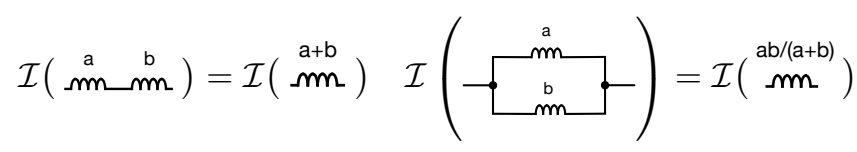

Proof.

$$
\mathcal{I}\left(\begin{array}{cc}
a & b \\
m & m
\end{array}\right)=a_{a b}
$$

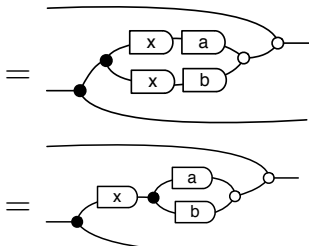$$
=\mathcal{I}\left(\begin{array}{c}
a+b \\
m
\end{array}\right)
$$
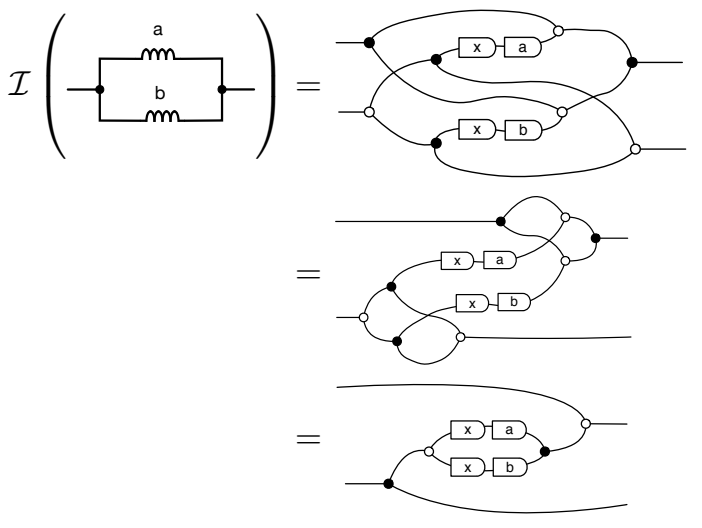

(Lemma 42)

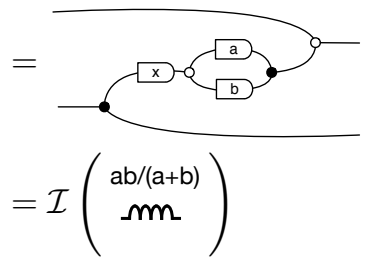


Lemma 47. [Properties of capacitors]

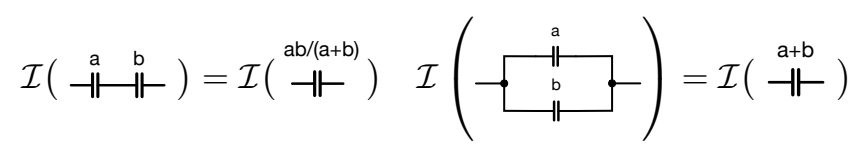

Proof.

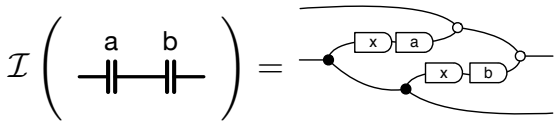

$$
\begin{aligned}
& =\sqrt{x-a-50} \\
& =\sqrt{a}-\frac{a}{b} \\
& =\mathcal{I}\left(\begin{array}{c}
\mathrm{ab} /(\mathrm{a}+\mathrm{b}) \\
\models
\end{array}\right)
\end{aligned}
$$

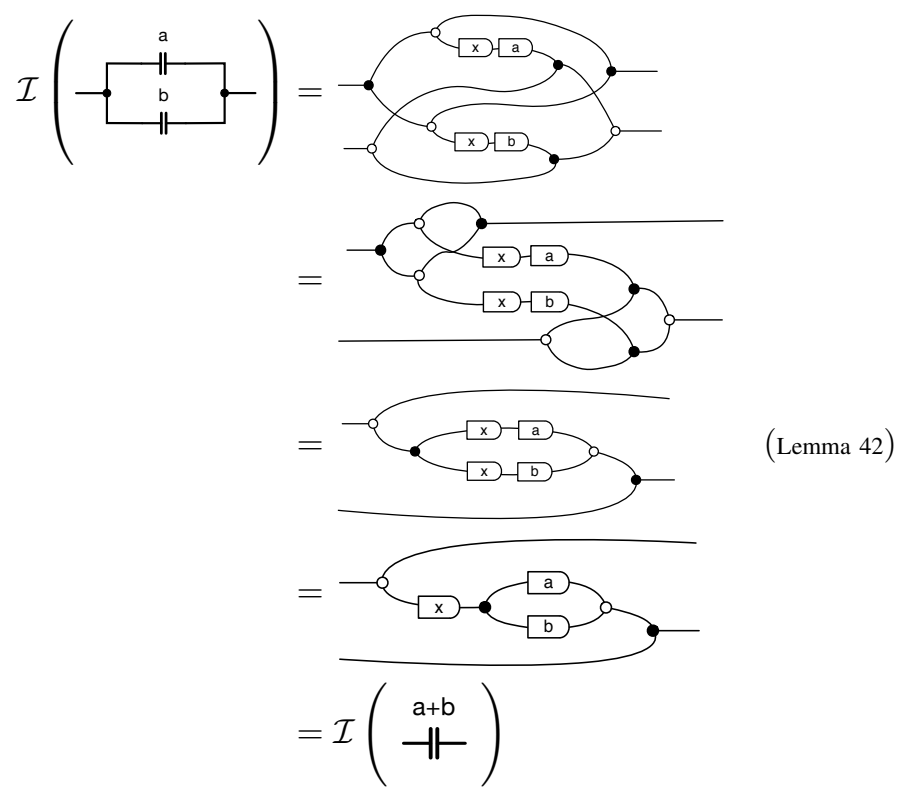

